\title{
New set of time-dependent Ginzburg-Landau equations for dirty superconductors near $T_{c}$
}

\author{
Chia-Ren $\mathrm{Hu}$ \\ Department of Phisics. Texas A\&M University. College Station. Texas 77843
}

(Received 30 July 1979)

\begin{abstract}
A critical reexamination is given to the microscopic derivation of a set of time-dependent Ginzburg-Landau (TDGL) equations, for dirty supercondutors near $T_{c}$ (not assuming gaplessness), by Kramer and Watts-Tobin and by Schön and Ambegaokar. The following improvements are first made: (i) A more general representation is used for the retarded $2 \times 2$ Green's function $\hat{G}^{R}$, valid in an arbitrary magnetic field; (ii) A "static approximation" for $\hat{G}^{R}$ is removed; (iii) An approximate gauge invariance is converted to an exact one, by some small changes in the equations for the order parameter and the charge density, in a way consistent with the approximation used. (iv) The simplifications of the collision integrals, based on a "mutilated-collision-operator" approximation, are shown to be also a direct consequence of the smallness of $1-T / T_{c}$. The set of equations is then tested against a local-charge-conservation law. A small violation is found, which leads to the discovery of an inconsistency in the truncation procedure used. Several new contributions are then found, which leads to a new set of TDGL equations. This contains all the above improvements, and also satisfies a local-chargeconservation law essentially exactly. At least some of the new contributions are shown to be important in the energy range of the order of the gap frequency.
\end{abstract}

\section{INTRODUCTION}

Ever since Gor'kov' obtained a microscopic derivation of the phenomenologically proposed static Ginzburg-Landau (GL) equations, ${ }^{2}$ based on his Green's-function formulation ${ }^{3}$ of the BCS theory, ${ }^{4}$ there has been a constant interest in and devotion to the generalization of the equations, so they could also be applied to time-dependent, nonequilibrium situations. Such a set of dynamic equations, in the form of a closed set of coupled partial differential equations for a smallest group of dynamic variables, has since been known as a set of "time-dependent GinzburgLandau" (TDGL) equations. This smallest group of dynamic variables must include the superconducting order parameter $\Delta$, the current and charge densities $\vec{j}$ and $\rho$, the scalar and vector potentials $\phi$ and $\vec{A}$, plus whatever new variables necessary to give a closed set of equations. A most ideal set of such equations should be subject to the only condition that limits the validity of the static GL equations, namely,

$|\Delta| \ll<$, or $1-T / T_{c} \ll<1$. It can then be valid for describing all intrinsic dynamic processes and instabilities in this temperature range, which may involve nonlinear deviations from equilibriuim, and any forms of spatial and/or temporal variations which might accompany the intrinsic processes. The equations should also be applicable to externally driven processes, but additional conditions must now be imposed, such as slow variation in time (i.e., with $\omega<<|\Delta|$ ), and in space (i.e., with $k<<T_{c} / V_{F}$, where $T_{c}$ is the transition temperature and $V_{F}$ the Fermi velocity). (Throughout this paper, we have used units with $\hbar=c=k_{B}=1$.)

The above goal turns out to be a very difficult one. The first attempt, made by Abrahams and Tsuneto ${ }^{5}$ more than a decade ago, was later realized to be valid only for an infinitesimal $|\Delta|$. A semiphenomenological set of TDGL equations was proposed by Schmid, ${ }^{6}$ who introduced for the first time the electrochemical potential $\psi$ as an additional dynamic variable, which is determined by the local-charge-conversation law $\dot{\rho}+\vec{\nabla} \cdot \vec{j}=0$. This gives the first clue that among the set of dynamic variables one must include some degrees of freedom that are associated with the excited quasiparticles which play the role of a normal fluid. The first major breakthrough on the microscopic derivation of a set of TDGL equations was made by Gor'kov and Éliashberg. ${ }^{7}$ They identified the difficulty in achieving this task with the singular nature of the BCS density of states at the gap frequency, ${ }^{4}$ and avoided it by restricting themselves to gapless superconductors containing a high concentration of magnetic impurities, so that $\tau_{s} T_{c}<<1$, where $\tau_{s}$ is the spin-flip-scattering lifetime of the electrons. They obtained the first consistent set of microscopically derived TDGL equations valid for a finite $|\Delta|$. This set of equations closely resembles the semiphenomenological set proposed by Schmid, ${ }^{6}$ and supports the notion that $\psi$ must be included in the minimum set of dynamic variables. Generalization of this derivation to low concentrations of magnetic impurities was made by Éliashberg, ${ }^{8}$ who still assumed a gaplessness condition $\tau_{s}|\Delta|<<1$. But in this case, the set of dynamic variables had to become greatly expanded: Two unknown functions of energy 
$\Gamma_{\epsilon}^{+} \pm \Gamma_{\epsilon}^{-}$, representing an infinite number of degrees of freedom, had to be introduced, in order to describe the behavior of the "normal electrons." These function were found to satisfy diffusion-type equations, but with complicated energy-dependent source terms which involved $\Delta, \phi$, and $\vec{A}$. (For a summary of this set of equations, generalized to allow for an arbitrary amount of magnetic and nonmagnetic scatterings, see Ref. 9.) Applications of this set of equations to physical problems gradually revealed that the functions $\Gamma_{\epsilon}^{+} \pm \Gamma_{\epsilon}^{-}$are closely related to the quasiparticle distribution function (more precisely, the even and odd parts of it, with respect to the energy variable $\epsilon$, which is measured with respect to the Fermi energy $\epsilon_{F}$ ). It then became increasingly clear that the ultimate set of dynamic variables should simply be $\Delta, \rho, \vec{j}, \phi$, and $\vec{A}$ and two distribution functions $f_{1}(\epsilon, \overrightarrow{\mathrm{R}}, t)$ and $\delta f(\epsilon, \overrightarrow{\mathrm{R}}, t)$ (which are even and odd with respect to $\epsilon$, respectively).

Intense efforts were made in the past few years to find the correct kinetic equations for these two distribution functions, from the microscopic theory for a finite-gap superconductor. The greatest successes were achieved by Schmid and Schön, ${ }^{10}$ and by Larkin and Ovchinnikov. ${ }^{11-13}$ When the above task was completed, the time became ripe again for deriving microscopically a closed set of TDGL equations, this time for a superconductor with a finite gap. Indeed, a set of such TDGL equations, which comes very close to the ideal goal outlined at the outset of this Introduction, has recently been presented by Kramer and Watts-Tobin ${ }^{14}$ for dirty superconductors near $T_{c}$. It still requires, besides $|\Delta| \ll T$, the additional assumption of a very short electron lifetime $\tau$ due to scattering by nonmagnetic impurities, (the meaning of "dirty"), that is $\tau T_{c}<1$. However, this is not much of a practical limitation, and involves no inprinciple difficulty for its removal. There do remain some major and minor deficiencies in this set of TDGL equations, however, the removal of which should be desirable: (i) Their equations for the spectral functions $N_{1}, R_{1}, N_{2}$, and $R_{2}$ are derived under the "static approximation," which is valid only for small deviations from a global equilibrium. (ii) Two of their equations are only approximately gauge invariant. It is important that these equations be modified so they can become exactly gauge invariant, since a non-gauge-invariant error can in principle be blown up to any size, simply by a suitable change of gauge. (iii) They have reduced the collision integrals in the kinetic equations for $\delta f$ and $f_{1}$, by invoking an approximation scheme introduced recently by Eckern and Schön, ${ }^{15}$ dubbed "mutilated collision operators." While this approximation scheme is based on sound physical principles, it is still more desirable to justify the reductions in terms of the single small parameter $|\Delta| / T$, for the set of TDGL equations derived. (iv) A special representation is used for the retarded ma- trix Green's function $\hat{G}^{R}$ as defined by Usadel, ${ }^{16}$ in terms of two complex quantities $\alpha$ and $\beta$. The representation is, strictly speaking, not valid in an arbitrary magnetic field. A generalization to the most general representation is desirable, even though in practical situations the special form is often sufficient.

Essentially the same set of TDGL equations has also been derived recently by Schön and Ambegaokar, ${ }^{17}$ except that they have only considered linear deviations from an equilibrium state, or a nondissipative steady state. No attempt was made by them to remove the deficiencies listed above, which also apply to their set of equations, if those equations are regarded as being derived for a general purpose.

In this paper, I have made a careful reexamination on the microscopic derivation of the set of TDGL equations obtained in Refs. 14 and 17. A systematic truncation procedure based on the smallness of $|\Delta| / T, \omega /|\Delta|, D k^{2} /|\Delta|, \delta f$, and $f_{1}$ is first found, which allows me to reproduce that set of equations. Improvements are then made on the derivation in order to remove all of the four deficiencies listed above. I then subject the resultant set of equations to an important test: namely, checking its satisfaction of a local-charge-conservation law. A small violation of this law $(\dot{\rho}+\vec{\nabla} \cdot \vec{j}=0)$ is found, which may or may not be regarded as serious. However, it has led me to the discovery that the truncation procedure used so far is not yet a consistent one, as judged from the viewpoint of an order analysis. This possibility arises because of the existence of two important energy scales $|\Delta|$ and $T$ in the present problem. Thus the smallness of $\delta f$ and $f_{1}$ in comparison with $f_{0}$ (which can be verified self-consistently), is not shared by $\partial(\delta f) / \partial \epsilon$ and $\partial f_{1} / \partial \epsilon$, in comparison with $\partial f_{0} / \partial \epsilon$, in the energy range $\epsilon \leq|\Delta|$. This point would not have important consequences, had it not been for the singular nature of the spectral functions at the gap frequency, which in some places makes the energy range $\epsilon \leq|\Delta|$ more important than the energy range $\epsilon \sim T$. This observation leads me to the discovery of a whole class of new contributions, the meaning of some of which I still do not fully understand. However, by introducing an approximation to include only the relatively more important terms among these new contributions, I obtain a new set of TDGL equations which is devoid of the deficiencies listed before, and satisfies a gauge invariance exactly, and the local-charge-conservation law essentially exactly. The difference between this new set of TDGL equations and the set obtained in Refs. 14 and 17 may be demonstrated to be important, except in special limiting situations.

This paper is organized as follows: In Sec. II, a brief review is given of a systematic approach developed by Larkin and Ovchinnikov ${ }^{11-13}$ for studying dynamic properties of superconductors. This approach combines the merits of a general Green's- 
function method for nonequilibrium processes as developed by Keldysh ${ }^{18}$ and Eilenberger's ${ }^{19}$ idea of using "energy-integrated Green's functions" to formulate a theory of superconductivity. A set of basic equations appears at the end of this review, which represents the basis of our microscopic derivation of a set of TDGL equations. In Sec. III, I proceed to review an approximation scheme developed by Larkin and Ovchinnikov, ${ }^{11-13}$ which is based on a theory of Usadel ${ }^{16}$ for dirty superconductors. As a result of this approximation, the set of basic equations obtained in Sec. II is further reduced to a form suitable for studying superconductors with a very short mean-free-path I. In Secs. IV and VII, I begin the process of reproducing the microscopic derivation of the set of TDGL equations obtained in Refs. 14 and 17 , and at the same time improving it, in order to remove the four deficiencies in this set of equations, as mentioned earlier in this Introduction. More precise$l y$, in Sec. IV, I analyze the normalization condition for $\hat{G}^{R}$ without assuming the static approximation, in order to deduce its most general representation. In Sec. $\mathrm{V}$, the equations for six spectral functions $N_{i}$ and $R_{i}$ (with $i=1-3$ ) are derived under this general representation, again not restricted to the static approximation. In Sec. VI, the kinetic equations for $\delta . f$ and $f_{1}$ are obtained, and shown to have an exact gauge invariance. It is pointed out here that the collision integrals in the equations for $\delta f$ and $f_{1}$ may be reduced to the desired forms, without invoking the concept of "mutilated collision operators." But the proof is deferred to Appendix B. In Sec. VII, expressions for $\Delta, \rho$, and $\vec{j}$ are obtained by the same procedure. The expressions for $\Delta$ and $\rho$ are found to be only approximately gauge invariant, so suggestions are made to alter them, in a way consistent with the approximation scheme, in order to make them exactly gauge invariant. In both Secs. VI and VII, I have followed a systematic truncation procedure, which is capable of reproducing all the terms kept by Refs. 14 and 17 , without attempting to find any new contributions (yet). In Sec. VIII, I have examined the set of TDGL equations obtained so far against the satisfaction of a local charge conservation law. It is found here that this law $\dot{\rho}+\vec{\nabla} \cdot \vec{j}=0$, is slightly violated, which may or may not be regarded as negligible. The presence of this marginal violation had let me to discover, in Sec. IX, an inconsistency in the truncation procedure used so far (with respect to an order analysis), which reveals the existence of several new contributions that are likely to be as important as the terms kept so far. Some such terms seem to require a complicated revision of the gauge property of $f_{1}$ (and possibly of $\delta f$ too), which is only partially understood. Fortunately, a certain combination involving $f_{1}$ is actually smaller than its presumed order needed for the truncation procedure used so far. An additional approximation can therefore be made, which gives rise to a new set of TDGL equations that is devoid of all the deficiencies present in the TDGL equations derived before, and satisfies a gauge invariance exactly, and the local charge conservation law essentially exactly. This new much enlarged set of TDGL equations has been summarized in Sec. X, together with a simplified version of it under two more independent approximations (both justified). This final set of equations now appears quite close to the set obtained in Refs. 14 and 17, but some essential differences remain, because some of the new contributions survive all of the approximations made so far. Finally, in closing this last section, an explicit demonstration is given of the significance of one of the new terms kept in the final set of TDGL equations, showing that the new terms do make the set of equations more physical. Two Appendixes have been attached at the end of this paper. Appendix A gives the detailed proof of an equation useful only in an intermediate step of the whole procedure. Appendix B provides a rigorous basis for the claim made in this paper that the "mutilated-collision-operator" approximation is a natural consequence of the small parameter $|\Delta| / T$ in the Ginzburg-Landau regime.

\section{BASIC EQUATIONS}

In the past few years, Larkin and Ovchinnikov (LO) ${ }^{11-13}$ have developed a powerful new technique for studying nonequilibrium phenomena in inhomogeneous superconductors. This technique incorporates Keldysh's general Green's-function method for nonequilibrium processes, ${ }^{18}$ and Eilenberger's idea for using energy-integrated Green's functions for studying superconductivity. ${ }^{19}$ More recently, LO $^{12,13}$ have further improved their technique, by discovering a very convenient new prescription for introducing two distribution functions in their approach [see Eqs. (3.20) and (3.21) below], and also for deriving two kinetic equations for these distribution functions. As a result, this technique of $\mathrm{LO}$ has provided a very elegant and systematic approach for studying dynamic properties of inhomogeneous superconductors. In this paper, I shall use the LO technique to study the problem of deriving, on microscopic basis, a consistent set of TDGL equations for dirty superconductors near $T_{c}$. In order for this paper to be self-contained, I shall first briefly review in this section the LO technique, and the use of it to arrive at a set of basic equations, which form the starting equations for the present derivation of a set of TDGL equations. Those who are already familiar with the works of LO should therefore proceed directly to Eqs. (2.10)-(2.18).

In terms of Nambu's notation for a pseudospinal field operator for the electrons: $\Psi(\vec{r}, t)$ identical to the transpose of $\left(\psi_{\uparrow}(\vec{r}, t), \psi_{i}^{\dagger}(\vec{r}, t)\right)$, three $2 \times 2$ ma- 
trix Green's functions are first defined for a superconductor (generalizing the original idea of Keldysh):

$\hat{G}^{R}\left(x, x^{\prime}\right) \equiv-i \hat{\tau}_{z}\left\langle\left[\Psi(x), \Psi^{\dagger}\left(x^{\prime}\right)\right]_{+}\right\rangle \Theta\left(t-t^{\prime}\right)$,

$\hat{G}^{A}\left(x, x^{\prime}\right) \equiv+i \hat{\tau}_{z}\left\langle\left[\Psi(x), \Psi^{\dagger}\left(x^{\prime}\right)\right]_{+}\right\rangle \Theta\left(t^{\prime}-t\right)$,

$\hat{G}^{K}\left(x, x^{\prime}\right) \equiv-i \hat{\tau}_{z}\left\langle\left[\Psi(x), \Psi^{\dagger}\left(x^{\prime}\right)\right]_{-}\right\rangle$,

where \langle\rangle denotes averaging over a thermal ensemble at $t=-\infty, x \equiv(\overrightarrow{\mathrm{r}}, t),[A, B]_{ \pm} \equiv A B \pm B A$, and $\left(\hat{\tau}_{x}, \hat{\tau}_{y}, \hat{\tau}_{z}\right)$ are the Pauli matrices. Following Keldysh ${ }^{18}$ and $\mathrm{LO}^{12,13}$ the three Green's functions defined in Eq. (2.1) are then used to define a single $4 \times 4$ matrix Green's function:

$$
\underline{G}\left(x, x^{\prime}\right) \equiv\left(\begin{array}{cc}
\hat{G}^{R}\left(x, x^{\prime}\right) & \hat{G}^{K}\left(x, x^{\prime}\right) \\
0 & \hat{G}^{A}\left(x, x^{\prime}\right)
\end{array}\right) .
$$

This $4 \times 4$ Green's function obeys essentially the same type of Feymann rules as the usual thermal Green's functions, except that a special connection matrix (defined in the two-dimensional "Keldysh space") must be used in association with each bare vertex. The connection matrix for an impurity or external-field vertex is just the trivial identity matrix, but for an electron-phonon vertex it is nontrivial: Thus the $4 \times 4$ electron self-energy due to the electron's interaction with a phonon field is given by (in the Migdal approximation)

$$
\left[\underline{\Sigma}^{\mathrm{ph}}\left(x x^{\prime}\right)\right]_{i j}=i g^{2} \tilde{\gamma}_{i i^{\prime}}^{k} \underline{G}_{i^{\prime} j^{\prime}}\left(x, x^{\prime}\right) \gamma_{j^{\prime} j}^{k^{\prime}} \underline{D}_{k^{\prime} k}\left(x^{\prime}, x\right),
$$

where $g$ is the electron-phonon coupling constant; all repeated indices here are summed over 1 and 2 in the Keldysh space, $\gamma_{i j}^{1}=\tilde{\gamma}_{i j}^{2}=2^{-1 / 2} \delta_{i j}, \gamma_{i j}^{2}=\tilde{\gamma}_{i j}^{1}$ $=2^{-1 / 2}\left(\tau_{x}\right)_{i j}$; and the $2 \times 2$ phonon propagator $\underline{D}$ is defined in terms of $D^{R}, D^{A}$, and $D^{K}$ in a way analogous to Eqs. (2.2) and (2.1), except that $[A, B]_{-}$and $[A, B]+$ are interchanged.

It is then straightforward to establish that for a weak-coupling superconducting system containing magnetic and nonmagnetic impurities, and interacting with an external electromagnetic field as well as a phonon bath (held at an equilibrium state of temperature $T$ ),

$$
\begin{aligned}
\underline{G}_{0}^{-1}(x) \underline{G}\left(x, x^{\prime}\right)= & \int d^{4} x_{1} \underline{\Sigma}\left(x, x_{1}\right) \underline{G}\left(x_{1}, x^{\prime}\right) \\
& +\delta^{4}\left(x-x^{\prime}\right),
\end{aligned}
$$

where

$$
\begin{aligned}
& \underline{G}_{0}^{-1}(x) \equiv i \underline{\tau}_{z} \partial_{t}-\epsilon\left(-i \vec{\nabla}-e \overrightarrow{\mathrm{A}} \underline{\tau}_{z}\right)+\mu-e \phi+\underline{\Delta}, \\
& \underline{\tau}_{z} \equiv\left(\begin{array}{ll}
\hat{\tau}_{z} & 0 \\
0 & \hat{\tau}_{z}
\end{array}\right), \quad \underline{\Delta} \equiv\left(\begin{array}{ll}
\hat{\Delta} & 0 \\
0 & \hat{\Delta}
\end{array}\right) \text { with } \hat{\Delta} \equiv\left(\begin{array}{cc}
0 & \Delta \\
-\Delta^{*} & 0
\end{array}\right), \\
& \underline{\Sigma}=\underline{\Sigma}^{\mathrm{nm}}+\underline{\Sigma}^{\mathrm{mag}}+\underline{\Sigma}^{\mathrm{ph}},
\end{aligned}
$$

where $\underline{\Sigma}^{\text {ph }}$ is given by Eq. (2.3), and $\underline{\Sigma}^{\mathrm{nm}}, \underline{\Sigma}^{\mathrm{mag}}$ are trivially obtainable from the equilibrium formalism (cf. Ref. 20) by replacing the electron thermal Green's function by $\underline{G}$.

Realizing that $G\left(x, x^{\prime}\right)$ and $\Sigma\left(x, x^{\prime}\right)$ are slowly varying functions of $\frac{1}{2}\left(\vec{r}+\vec{r}^{\prime}\right)$ [length scale: $\left.\xi(T) \sim \hbar v_{F} /|\Delta|\right]$, and rapidly varying functions of $\left(\vec{r}-\vec{r}^{\prime}\right)$ [length scale: $\hbar / P_{F}<<\xi(T)$ ], one may approximate Eq. (2.4) by ${ }^{19}$

$$
\left[i \underline{\tau}_{z} \partial_{t}-\xi-\overrightarrow{\mathrm{v}}_{F} \cdot\left(-\frac{1}{2} i \vec{\nabla}_{R}-e \overrightarrow{\mathrm{A}} \underline{\tau}_{z}\right)-e \phi+\underline{\Delta}\right] \underline{G}\left(t, t^{\prime}, \overrightarrow{\mathrm{P}}, \overrightarrow{\mathrm{R}}\right)=\int d t_{1} \underline{\Sigma}\left(t, t_{1}, \overrightarrow{\mathrm{P}}, \overrightarrow{\mathrm{R}}\right) \underline{G}\left(t_{1}, t^{\prime}, \overrightarrow{\mathrm{P}}, \overrightarrow{\mathrm{R}}\right)+\delta\left(t-t^{\prime}\right)
$$

where

$$
\xi \equiv \epsilon(\overrightarrow{\mathrm{P}})-\mu, \quad \overrightarrow{\mathrm{v}}_{F} \equiv \partial \epsilon /\left.\partial \overrightarrow{\mathrm{P}}\right|_{P-P_{F}},
$$

and

$$
\underline{G}\left(t, t^{\prime}, \overrightarrow{\mathrm{P}}, \overrightarrow{\mathrm{R}}\right) \equiv \int d^{3} s e^{-i \overrightarrow{\mathrm{P}} \cdot \overrightarrow{\mathrm{s}}} \underline{G}\left(t, t^{\prime}, \overrightarrow{\mathrm{R}}+\frac{1}{2} \overrightarrow{\mathrm{s}}, \overrightarrow{\mathrm{R}}-\frac{1}{2} \overrightarrow{\mathrm{s}}\right) .
$$

It is then straightforward to eliminate the $\xi$ term by using a conjugate equation. An equation for the energyintegrated Green's function:

$$
\underline{G}_{\hat{P}}\left(t, t^{\prime}, \overrightarrow{\mathrm{R}}\right) \equiv \frac{i}{\pi} \int d \xi \underline{G}\left(t, t^{\prime}, \overrightarrow{\mathrm{P}}, \overrightarrow{\mathrm{R}}\right),
$$

can then be easily obtained, which reads

$$
v_{F} \hat{P} \cdot \vec{\nabla} \underline{\mathrm{R}}_{\hat{P}}+\underline{\tau}_{z} \partial_{t} \underline{G}_{\hat{P}}+\partial_{t^{\prime}} \underline{G}_{\hat{P}} \underline{\tau}_{z}+\underline{H}(t) \underline{G}_{\hat{P}}-\underline{G}_{\hat{P}} \underline{H}\left(t^{\prime}\right)+i \int_{-\infty}^{\infty} d t_{1}\left[\underline{\Sigma}_{\hat{P}}\left(t, t_{1}\right) \underline{G}_{\hat{P}}\left(t_{1}, t^{\prime}\right)-\underline{G}_{\hat{P}}\left(t, t_{1}\right) \underline{\Sigma}_{\hat{P}}\left(t, t^{\prime}\right)\right]=0,
$$

where $\hat{P} \equiv \overrightarrow{\mathrm{P}} /|\overrightarrow{\mathrm{P}}|$,

$$
\underline{H}(t) \equiv-i e v_{F} \hat{P} \cdot \overrightarrow{\mathrm{A}} \underline{\tau}_{z}-i \underline{\Delta}+i e \phi,
$$


and some arguments like $\vec{R}, t$, and/or $t^{\prime}$, have been suppressed in these equations for the sake of clarity. In obtaining Eq. (2.10), use has been made of the fact that to a very good approximation, the selfenergy $\underline{\Sigma}$ depends only on the direction of $\overrightarrow{\mathbf{P}}$ (hence the notation $\left.\underline{\Sigma}_{\hat{p}}\right),{ }^{20}$ can be expressed in terms of $\underline{G}_{\hat{p}}$ :

$$
\begin{aligned}
& \underline{\Sigma}_{\hat{P}}=\underline{\Sigma}_{\hat{P}}^{n m}+\underline{\Sigma}_{\hat{P}}^{\text {mag }}+\underline{\Sigma}_{\hat{P}}^{\text {ph }}, \\
& \underline{\Sigma}_{\hat{P}}^{n m}=-\frac{1}{2} i n v_{F} \int d \Omega_{\hat{P}_{1}} \sigma_{\hat{P}, \hat{P}_{1}} \underline{G}_{\hat{P}_{1}}\left(t, t^{\prime}, \overrightarrow{\mathrm{R}}\right), \\
& \underline{\Sigma}_{\hat{P}}^{\text {mag }}=-\left(i / 8 \pi \tau_{s}\right) \int d \Omega_{\hat{P}_{1}} \underline{\tau}_{z} \underline{G}_{\hat{P}_{1}} \underline{\tau}_{z},
\end{aligned}
$$

(where $n$ is the electron density, $\tau_{s}$ is the spin-flip lifetime, with only $s$-wave spin-flip scattering included), and

$$
\underline{\Sigma}_{\hat{P}}^{p h}=\left(\begin{array}{cc}
\hat{\Sigma}_{\hat{P}}^{p h, R} & \hat{\Sigma}_{\hat{P}}^{p h, K} \\
0 & \hat{\Sigma}_{\hat{p}}^{p h, A}
\end{array}\right)
$$

where

$$
\begin{aligned}
& \hat{\Sigma}_{\hat{P}}^{\mathrm{ph}, R(\operatorname{or} A)}=\frac{1}{8} N(0) g^{2} \int d \Omega_{\hat{P}_{1}}\left[D_{\hat{P}_{1}}^{K} \cdot \hat{P}\left(t^{\prime}-t\right) \hat{G}_{\hat{P}_{1}}^{R(\operatorname{or} A)}\left(t, t^{\prime}\right)+D_{\hat{P}_{1} \cdot \hat{P}}^{A(\operatorname{or} R)}\left(t^{\prime}-t\right) \hat{G}_{\hat{P}_{1}}^{K}\left(t, t^{\prime}\right)\right], \\
& \hat{\Sigma}_{\hat{P}}^{\mathrm{ph}, K}=\frac{1}{8} N(0) g^{2} \int d \Omega_{\hat{P}_{1}}\left\{D_{\hat{P}_{1} \cdot \hat{P}}^{K}\left(t^{\prime}-t\right) \hat{G}_{\hat{P}_{1}}^{K}\left(t, t^{\prime}\right)-\left[D_{\hat{P}_{1} \cdot \hat{P}}^{R}\left(t^{\prime}-t\right)-D_{\hat{P}_{1}}^{A} \cdot \hat{P}\left(t^{\prime}-t\right)\right]\left[\hat{G}_{\hat{P}_{1}}^{R}\left(t, t^{\prime}\right)-\hat{G}_{\hat{P}_{1}}^{A}\left(t, t^{\prime}\right)\right]\right\},
\end{aligned}
$$

where $N(0)$ is the normal electronic density of states at the Fermi surface.

Equations $(2.10)-(2.17)$, plus the normalization condition

$$
\int_{-\infty}^{\infty} d t_{1} \underline{G}_{\hat{p}}\left(t, t_{1}\right) \underline{G}_{\hat{p}}\left(t_{1}, t^{\prime}\right)=\underline{1} \delta\left(t-t^{\prime}\right)
$$

constitute the complete set of basic equations obtained by LO. [See Ref. 12, Eqs. (4) and (6)-(8).] The normalization condition was first obtained by Eilenberger ${ }^{19}$ for the electronic thermal Green's function of a superconductor. It is extended to the Keldysh formalism by LO. ${ }^{11}$ I am not aware of any simple rigorous proof for this relation, so I will not give any, except the remark that it is consistent with Eq. (2.10), which will become more transparent below [cf. Eq. (3.2b) and the discussion made in the paragraph just above Eq. (3.4)].

\section{DIRTY-LIMIT APPROXIMATION}

The material presented in this section is also not new, but was originally developed by LO. ${ }^{11,12}$ It is reproduced here to make this paper self-contained. Besides, the compact notation introduced here will be useful in our derivation of TDGL. Those who are familiar with Refs. 11 and 12 may proceed to Eqs. (3.11)-(3.13), which should be read with the definitions given in Eqs. (3.1) and (3.4).

We start by introducing a very compact notation

$$
(\underline{A} \circ \underline{B})_{i j}\left(t, t^{\prime}\right) \equiv \sum_{k=1}^{4} \int_{-\infty}^{\infty} d t_{1} \underline{A}_{i k}\left(t, t_{1}\right) \underline{B}_{k j}\left(t_{1}, t^{\prime}\right) \text {, }
$$

$$
[\underline{A}, \underline{B}]^{\circ} \equiv \underline{A} \circ \underline{B}-\underline{B} \circ \underline{A} .
$$

Then Eqs. (10) and (18) may be recast into the very simple forms:

$$
\begin{aligned}
& {\left[\underline{Z}_{\hat{P}}, \underline{G}_{\hat{P}}\right]^{\circ}=0,} \\
& \underline{G}_{\hat{P}} \circ \underline{G}_{\hat{P}}=\underline{1},
\end{aligned}
$$

where 1 here includes a factor $\delta\left(t-t^{\prime}\right)$, and

$$
\begin{aligned}
\underline{Z}_{\hat{P}}\left(t, t^{\prime}\right) \equiv & {\left[v_{F} \hat{P} \cdot \vec{\nabla}_{\vec{R}}+\underline{\tau}_{z} \partial_{t}+\underline{H}(t)\right] \delta\left(t-t^{\prime}\right) } \\
& +i \underline{\Sigma}_{\hat{P}}\left(t, t^{\prime}\right)
\end{aligned}
$$

Note that $\underline{Z}_{\hat{p}}$ is an operator in $R$ space as well, so $\left[\vec{\nabla}_{\vec{R}}, f(\vec{R})\right]=\left[\vec{\nabla}_{\vec{R}} f(\vec{R})\right]$ must be understood. The notation $[,]^{\circ}$ has all the properties of a commutator, so from $\left[\underline{Z}_{\hat{P}}, \underline{G}_{\hat{P}}\right]^{\circ}=0$, one can deduce

$\left[\underline{Z}_{\hat{P}}, \underline{G}_{\hat{P}} \circ \underline{G}_{\hat{P}}\right]^{\circ}=0$. It is consistent with this equation to assume $\underline{G}_{\hat{P}} \circ \underline{G}_{\hat{P}}=$ const $\times \underline{1}$. The constant may then be "determined" as being unity, by imagining a nonequilibrium superconductor to be connected to an equilibrium superconductor (or normal metal) at $|\vec{R}|=\infty$. This argument roughly establishes the normalization condition, Eq. (3.2b). ${ }^{11}$

In the dirty limit, $\tau T_{c}<<1$, the following approximation may be used (due originally to Usadel ${ }^{16}$ ):

$$
\underline{G}_{\hat{P}} \simeq \underline{G}+\hat{P} \cdot \underline{\vec{G}}_{1} \quad\left(\text { with } \hat{P} \cdot \underline{\vec{G}}_{1}<<\underline{G}\right) .
$$

Note that in the $s-p$ approximation for nonmagnetic impurity scatterings,

$$
\underline{\boldsymbol{\Sigma}}_{\hat{P}}=\underline{\boldsymbol{\Sigma}}+\hat{P} \cdot \underline{\vec{\Sigma}}_{1}
$$

where

$$
\begin{aligned}
\underline{\Sigma} & =-(i / 2 \tau) \underline{G}-\left(i / 2 \tau_{s}\right) \underline{\tau}_{z} \underline{G} \underline{\tau}_{z}+\underline{\Sigma}^{p h} \\
& \equiv-(i / 2 \tau) \underline{G}+\underline{\Sigma}^{\prime}
\end{aligned}
$$


and

$$
\underline{\vec{\Sigma}}_{1} \simeq-(i / 2)\left(\tau^{-1}-\tau_{\mathrm{tr}}^{-1}\right) \underline{\overrightarrow{\mathrm{G}}}_{1} .
$$

( $\tau$ and $\tau_{\mathrm{tr}}$ denotes nonspin-flip isotropic and transport lifetimes, respectively.) Furthermore,

$$
\underline{Z}_{\hat{P}}=\underline{Z}+\hat{P} \cdot \underline{Z}_{1}
$$

where

$$
\underline{Z} \equiv(2 \tau)^{-1} \underline{G}+\underline{Z}^{\prime},
$$

with

$$
\underline{Z}^{\prime} \equiv\left(\underline{\hat{\tau}}_{z} \partial_{t}-i \Delta+i e \phi\right) \delta\left(t-t^{\prime}\right)+i \underline{\Sigma}^{\prime}\left(t, t^{\prime}\right)
$$

and

$$
\underline{\vec{z}}_{1} \equiv v_{F} \underline{\vec{\partial}}+i \underline{\vec{z}}_{1},
$$

where

$$
\underline{\partial}\left(t, t^{\prime}\right) \equiv\left(\vec{\nabla}_{\overrightarrow{\mathrm{R}}}-i e \overrightarrow{\mathrm{A}} \underline{\underline{\tau}}_{z}\right) \delta\left(t-t^{\prime}\right) .
$$

Thus Eq. (3.2a) may be split into two equations, by using angular averaging with and without a factor $\hat{P}$ inserted in the integrand. The two equations are

$$
\left[\underline{Z}^{\prime}, \underline{G}\right]^{\bullet}+\frac{1}{3} v_{F}\left[\underline{\Xi}, \underline{\vec{G}}_{1}\right]^{\bullet}=0
$$

(where a vector dot product is also implied in the second term), and

$$
\boldsymbol{v}_{\digamma}[\underline{\underline{\jmath}}, \underline{G}]^{\circ}+\frac{1}{2}\left(\tau^{-1}-\tau_{\mathrm{tr}}^{-1}\right)\left[\underline{\vec{G}}_{1}, \underline{G}\right]^{\circ}+\left[\underline{Z}, \underline{\vec{G}}_{1}\right]^{\circ}=0 .
$$

In view of Eq. (3.4), the normalization condition, Eq. $(3.2 b)$, is also reduced to

$$
\underline{G} \circ \underline{G}=\underline{1}, \quad \underline{G} \circ \underline{\vec{G}}_{1}+\underline{\vec{G}}_{1} \circ \underline{G}=0 .
$$

In order to be consistent with the dirty-limit approximation, the factor $\underline{Z}$ in the last term of Eq. (3.10b) should be replaced by its leading term, $(2 \tau)^{-1} \underline{G}$. Then Eq. (3.10b) may be solved with the help of Eq. (3.11), giving

$$
\underline{\mathrm{G}}_{1}=-l(\underline{G} \circ \underline{\partial} \circ \underline{G}-\underline{\partial}), \quad l \equiv v_{F} \tau_{\mathrm{tr}} .
$$

Substituting this equation back into Eq. (3.10a) gives

$$
\left[\left(\underline{Z}^{\prime}-D \underline{\partial} \circ \underline{G} \circ \underline{\partial}\right), \underline{G}\right]^{\circ}=0,
$$

where $D \equiv \frac{1}{3} v_{F} l$ is the diffusion constant.

Equations (3.12), (3.13), and the first part of Eq. (3.11) constitute the basic equations for a dirty superconductor first obtained by LO, but they are expressed here in a more compact set of notations. If the different elements of the matrix equations in the Keldysh space are separated out, this set of equations would read

$$
\begin{aligned}
& \hat{G}^{R} \circ \hat{G}^{R}=\hat{G}^{A} \circ \hat{G}^{A}=\hat{\imath}, \\
& \hat{G}^{R} \circ \hat{G}^{K}+\hat{G}^{K} \circ \hat{G}^{A}=0, \\
& \overrightarrow{\hat{G}}_{1}^{R(\operatorname{or} A)}=l\left(\hat{G}^{R(\text { or } A)} \circ \vec{\partial} \circ \hat{G}^{R(\operatorname{or} A)}-\vec{\partial}\right),
\end{aligned}
$$

where

$$
\vec{\partial}\left(t, t^{\prime}\right) \equiv\left(\vec{\nabla}_{R}-i e \overrightarrow{\mathrm{A}} \hat{\tau}_{z}\right) \delta\left(t-t^{\prime}\right) .
$$

Furthermore,

$$
\begin{aligned}
& \overrightarrow{\hat{G}}_{1}^{K}=l\left(\hat{G}^{R} \circ \vec{\partial} \circ \hat{G}^{K}+\hat{G}^{K} \circ \vec{\partial}_{\partial} \circ \hat{G}^{A}\right), \\
& {\left[\left(\hat{Z}^{\prime R(\operatorname{or} A)}-D \vec{\partial} \circ \hat{G}^{R(\text { or } A)} \circ \vec{\partial}\right), \hat{G}^{R(\text { or } A)}\right]^{\circ}=0}
\end{aligned}
$$

and

$$
\left(\hat{Z}^{\prime R}-D \vec{\partial} \circ \hat{G}^{R} \circ \vec{\partial}\right) \circ \hat{G}^{K}-\hat{G}^{K} \circ\left(\hat{Z}^{\prime A}-D \vec{\partial} \circ \hat{G}^{A} \circ \widehat{\partial}\right)+\left(\hat{Z}^{K}-D \vec{\partial} \circ \hat{G}^{K} \circ \vec{\partial}\right) \circ \hat{G}^{A}-\hat{G}^{R} \circ\left(Z^{K}-D \vec{\partial}^{\prime} \circ \hat{G}^{K} \circ \vec{\partial}\right)=0
$$

All matrices in Eqs. (3.14) -(3.19) are now defined in the Nambu (particle-hole) space only, and are therefore $2 \times 2$. Equations (3.1) are therefore used here with index-sum running from 1 to 2 .

$\mathrm{LO}^{12,13}$ then notices that Eq. (3.15) is exactly satisfied by putting

$$
\hat{G}^{K}=\hat{G}^{R} \circ \hat{f}-\hat{f} \circ \hat{G}^{A} .
$$

While even with an arbitrary $2 \times 2$ matrix $\hat{f}$, I know of no rigorous proof that Eq. (3.20) is the most general solution of Eq. (3.15), LO further restrict $\hat{f}$ to the form:

$$
\hat{f}=\left(f_{0}+\delta f\right) \hat{1}+f_{1} \hat{\tau}_{z}
$$

The two functions $\delta f\left(t, t^{\prime}, \overrightarrow{\mathbf{R}}\right)$ and $f_{1}\left(t, t^{\prime}, \overrightarrow{\mathrm{R}}\right)$ are then identified by LO as the deviations of the quasiparticle distribution function from equilibrium, corresponding to the longitudinal and transverse modes first discussed by Schmid and Schön. ${ }^{10}$ ( $f_{0}$ corresponds to an equilibrium distribution. See Sec. $\mathrm{V}$ for its value.) Equations (3.19) - (3.21) provide an extremely con- 
venient way for deriving the kinetic equations, although their justification still remains somewhat incomplete at the present time, which I shall not attempt to improve (except the remarks in Refs. 24 and 25).

Substituting Eq. (3.20) into Eq. (3.19) and using Eq. (3.18), one may obtain

$$
\hat{G}^{R} \circ \hat{Y}-\hat{Y} \circ \hat{G}^{A}=0 \text {. }
$$

where

$$
\begin{aligned}
\hat{Y} \equiv & \left(\hat{Z}^{\prime R} \circ \hat{f}-\hat{f} \circ \hat{Z}^{\prime A}-\hat{Z}^{\prime \kappa}\right) \\
& -D \widehat{\partial} \circ \hat{G}^{R} \circ[\overrightarrow{\hat{\partial}}, \hat{f}]^{\circ}-D[\overrightarrow{\hat{\partial}}, \hat{f}]^{\circ} \circ \hat{G}^{A} \circ \vec{\jmath}
\end{aligned}
$$

Equations (3.21)-(3.23) are used in Sec. $V$ to derive two coupled kinetic equations for $\delta f$ and $f_{1}$.

\section{GENERAL IMPLICATIONS OF THE NORMALIZATION CONDITION AT LOW FREQUENCIES}

To derive a consistent set of TDGL in the dirty limit, I first make the fundamental assumption that $\Delta<<T$, which is equivalent to $1-T / T_{c}<<1$. In a self-consistent argument, $\mathrm{I}$ then assume that all important intrinsic frequencies and wave numbers are such that $\omega$ and $D k^{2}$ are both no larger than the or$\operatorname{der} \eta|\Delta|$, where $\eta \equiv|\Delta| / T$. (These assumptions may be verified a posteriori by the set of TDGL equations derived, so they are not independent restrictions to the validity regime of the equations, unless the spatial and temporal variations are imposed externally.) Now under these assumptions, all functions of $t$ and $t^{\prime}$ introduced so far will be slowly varying functions of $\frac{1}{2}\left(t+t^{\prime}\right)$ (time scale $\left.\sim \omega^{-1}\right)$, and rapidly varying functions of $\left(t-t^{\prime}\right)$ (time scale $\sim T^{-1}$ or $\left.|\Delta|^{-1}\right)$. It is therefore convenient to define

$$
\begin{gathered}
A(\epsilon, \bar{l}) \equiv \int_{-\infty}^{\infty} d\left(t-t^{\prime}\right) A\left(\bar{t} \equiv \frac{t+t^{\prime}}{2}, t-t^{\prime}\right) \\
\times \exp \left[i \epsilon\left(t-t^{\prime}\right)\right] .
\end{gathered}
$$

and use the approximation introduced by $\mathrm{LO}^{12}$ :

$$
(A \circ B)(\epsilon, \bar{l})=A(\epsilon, \bar{l}) B(\epsilon, \bar{l})+\frac{1}{2} i\{A, B\}_{\epsilon, \bar{t}}
$$

where $\{A, B\}_{\epsilon, i}$-denotes a Poisson bracket:

$$
\{A, B\}_{\epsilon, \bar{t}} \equiv \frac{\partial A}{\partial \epsilon} \frac{\partial B}{\partial \tau}-\frac{\partial A}{\partial t} \frac{\partial B}{\partial \epsilon} .
$$

Applying Eq. (4.2) to the normalization conditions Eq. (3.14) (with $t$ replacing $\bar{l}$ ), and introducing the following generally valid representation

$$
\begin{aligned}
\hat{G}^{R(\text { or } A)}(\epsilon, t)= & \alpha^{R(\operatorname{or} A)} \hat{\tau}_{z}+\tilde{\beta}^{R(\text { or } A)} \hat{\tau}_{y} \\
& +\tilde{\gamma}^{R(\operatorname{or} A)} \hat{\tau}_{x}+\delta^{R(\operatorname{or} A)} \hat{1} .
\end{aligned}
$$

I obtain

$$
\begin{aligned}
& \alpha^{2}+\tilde{\beta}^{2}+\tilde{\gamma}^{2}+\delta^{2}=1 . \\
& 2 \alpha \delta=\{\tilde{\gamma}, \tilde{\beta}\}_{\epsilon, t} . \\
& 2 \tilde{\beta} \delta=\{\alpha, \tilde{\gamma}\}_{\epsilon, t} . \\
& 2 \tilde{\gamma} \delta=\{\tilde{\beta}, \alpha\}_{\epsilon, t} .
\end{aligned}
$$

where the superscript $R$ (or $A$ ) has been suppressed. Multiplying Eqs. $(4.5 \mathrm{~b})-(4.5 \mathrm{~d})$ by $\partial_{\epsilon} \alpha, \partial_{\epsilon} \tilde{\beta}$, and $\partial_{\epsilon} \tilde{\gamma}$, respectively, then adding up the three equations and using Eq. (4.5a), one gets $\delta \partial_{\epsilon}\left(\delta^{2}\right)=0$. Since $\delta=0$ for $|\epsilon|=\infty$, this equation implies $\delta \equiv 0$. [One may also derive the equation $\delta \partial_{t}\left(\delta^{2}\right)=0$, and then conclude that $\delta \equiv 0$, because it is true at $t=-\infty$.]

Actually, because we have used the approximation in Eq. (4.2), we have only established that $\delta$ vanishes to the nominal order $\omega / \Delta \sim \eta$. (In this regard, $\alpha, \tilde{\beta}$, and $\tilde{\gamma}$ will be taken as quantities of order unity, and $\epsilon$ as of order $\Delta$. See further discussion of this point in Sec. IX and in particular, Ref. 26 below.) It seems that this conclusion is already suffficient for our derivation of a set of TDGL equations in the low(intrinsic-) frequency regime. I therefore conclude that Eq. (4.4) without the $\delta$ term is actually valid bevond the static approximation assumed by previous investigations. ${ }^{14,17}$

Now in the previous derivations of a set of TDGL equations ${ }^{14,17}$ it was actually assumed that

$$
\hat{G}^{R(\text { or } A)}=\alpha^{R(\text { or } A)} \hat{\tau}_{z}+\beta^{R(\text { or } A)} \hat{\tau}_{\chi} .
$$

where $\hat{\tau}_{x} \equiv \cos \chi \hat{\tau}_{y}+\sin \chi \hat{\tau}_{x}$, with $\chi$ being the phase of the order parameter, $\Delta=|\Delta| \exp (i \chi)$. Equation (4.6) has been introduced previously by Schmid and Schön, and is rigorously valid for a static superconductor in the absence of an external magnetic field. References 14 and 17 assumed Eq. (4.6), because they assumed that the equations for $\hat{G}^{R(\text { or } A)}$ may be obtained in the static approximation, and the main problems of their concern are indeed at zero external field. Actually, a static approximation for $\hat{G}^{R(\operatorname{or} A)}$ is strictly valid only for linear deviations from equilibrium (which is the main concern of Ref. 17, but not of Ref. 14). The approximation should therefore be avoided in our derivation of a general set of TDGL equations, which are supposed to be also applicable to intrinsic nonlinear processes and instabilities. Also, allowing for an arbitrary external magnetic field is certainly a desirable feature in the set of TDGL equations to be derived. Not knowing whether Eq. (4.6) is still valid exactly or in any approximate sense under these general conditions, I shall replace Eq. (4.6) by

$$
\hat{G}^{R(\text { or } A)}=\alpha^{R(\text { or } A)} \hat{\tau}_{z}+\beta^{R(\text { or } A)} \hat{\tau}_{X}+\gamma^{R(\text { or } A)} \hat{\tau}_{X+\pi / 2} .
$$


where

$$
\hat{\tau}_{\chi+\pi / 2} \equiv-\sin \chi \hat{\tau}_{y}+\cos \chi \hat{\tau}_{x}
$$

The relation between Eqs. (4.7) and (4.4) is then

$$
\begin{aligned}
& \tilde{\beta}=\beta \cos \chi-\gamma \sin \chi, \\
& \tilde{\gamma}=\beta \sin \chi+\gamma \cos \chi,
\end{aligned}
$$

which may also be written as

$$
\tilde{\beta} \pm i \tilde{\gamma}=\exp ( \pm i \chi)(\beta \pm i \gamma) \text {. }
$$

Equation (4.9) will be very convenient for transforming the TDGL equations derived from a general gauge into the gauge in which $\Delta$ appears as $|\Delta|$ (henceforth called the real- $\Delta$ gauge). I emphasize that $\beta$, $\tilde{\beta}, \gamma$, and $\tilde{\gamma}$ are all complex quantities, so the reader should not be misled by Eq. (4.9).

After I have derived all the equations, I shall return to the validity of Eq. (4.6), and show that it is actually a valid approximation to assume Eq. (4.6) for the general situation under consideration, provided that this approximation is introduced consistently, and one is not interested in studying any physical phenomena which might be caused solely by the presence of a small but nonvanishing $\underline{\gamma}$.

\section{DERIVATION OF EQUATIONS FOR THE SPECTRAL FUNCTIONS}

For this purpose, we first apply Eqs. (4.1) and (4.2) to Eq. (3.18), which gives

$$
\left[\hat{Z}^{\prime R(\operatorname{or} A)}-D \vec{\partial} \cdot\left(\hat{G}^{R(\text { or } A)} \vec{\partial}\right), \hat{G}^{R(\operatorname{or} A)}\right]+\frac{1}{2} i\left\{\hat{Z}^{\prime R(\operatorname{or} A)}, \hat{G}^{R(\operatorname{or} A)}\right\}_{\epsilon, t}-\frac{1}{2} i\left\{\hat{G}^{R(\operatorname{or} A)}, \hat{Z}^{\prime R(\operatorname{or} A)}\right\}_{\epsilon, t}=0
$$

where $[\hat{A}, \hat{B}]$ stands simply for $\hat{A} \hat{B}-\hat{B} \hat{A}$, and

$$
\begin{aligned}
\hat{Z}^{\prime R(\operatorname{or} A)}(\epsilon, t)= & -i \epsilon \hat{\tau}_{z}-i \hat{\Delta}+i e \phi+\left(2 \tau_{s}\right)^{-1} \hat{\tau}_{z} \hat{G}^{R(\operatorname{or} A)} \hat{\tau}_{z} \\
& +\frac{1}{8} i N(0) g^{2} \int d \Omega_{\hat{P}_{1}} \int \frac{d \epsilon_{1}}{2 \pi}\left[D_{\hat{P}_{1}}^{K} \cdot \hat{P}\left(\epsilon_{1}-\epsilon\right) \hat{G}^{R(\operatorname{or} A)}\left(\epsilon_{1}\right)+D_{\hat{P}}^{A}(\operatorname{or} R)\left(\epsilon_{1}-\epsilon\right) \hat{G}^{K}\left(\epsilon_{1}\right)\right] .
\end{aligned}
$$

In the reduction of Eq. (3.18) to Eq. (5.1), I have consistently neglected terms quadratic in $\omega$ and/or $D k^{2}$. While a rigorous justification of this procedure is difficult in view of the singular nature of $\hat{G}^{R}$ and $\hat{G}^{A}$ at the gap frequency, ${ }^{21}$ I point out that this procedure should be at least more accurate than the "static approximation" used by Kramer and WattsTobin, which would mean that the last two terms in Eq. (5.1) are also neglected. As I have already remarked in Sec. IV, a static approximation for $\hat{G}^{R(\text { or } A)}$ is valid only for small deviations from a true equilibrium, and is therefore unsatisfactory for the present purpose. On the other hand, the set of TDGL equations derived here corresponds to only small deviations from a local equilibrium. The kinetic equations for $\delta f$ and $f_{1}$ that $I$ obtain in the next section do appear to be linearized, but this is due to the assumption that $|\Delta| / T$ is small, from which one may deduce that $\delta f$ and $f_{1}$ are also small. Thus in nowhere of this work, have I restricted the derivation to only linear dynamic processes.

To proceed, I first take into account an observation made by $\mathrm{LO}^{12}$ that the last term in Eq. (5.2), which is important only for $\epsilon \sim|\Delta|$, may be simplified under the assumption $|\Delta|<<T$. If we assume that the phonons are in thermal equilibrium at a temperature
$T$, then $^{11}$

$$
\begin{aligned}
& D \frac{R}{\mathrm{k}}(\omega)=\left[D \frac{A}{\mathrm{k}}(\omega)\right]^{*}=-\frac{\omega^{2}(\overrightarrow{\mathrm{k}})}{\omega^{2}(\overrightarrow{\mathrm{k}})-(\omega+i \delta)^{2}}, \\
& D \frac{K}{\mathrm{k}}(\omega)=\operatorname{coth}(\omega / 2 T)\left[D \frac{R}{\mathrm{k}}(\omega)-D \frac{A}{\mathrm{k}}(\omega)\right] .
\end{aligned}
$$

If we also neglect virtual processes in Eq. (5.2) due to $D^{R}+D^{A}$, and keep only real "lifetime" processes due to

$$
\begin{aligned}
\left(D^{R}-D^{A}\right)_{\overrightarrow{\mathrm{k}}}(\omega)= & -i \pi \omega(\overrightarrow{\mathrm{k}}) \\
& \times[\delta(\omega-\omega(\overrightarrow{\mathrm{k}}))-\delta(\omega+\omega(\overrightarrow{\mathrm{k}}))]
\end{aligned}
$$

(which is a standard approximation for weak coupling superconductors), then the dominant contribution to the last term in Eq. (5.2) will come from the integration region where $\left.\omega(\overrightarrow{\mathrm{k}})\right|_{\overrightarrow{\mathrm{k}}-\overrightarrow{\mathrm{P}}_{1}-\overrightarrow{\mathrm{P}}} \simeq C_{0} P_{F} \theta \sim T\left[C_{0}\right.$ being the speed of sound, $\theta \equiv \cos ^{-1}\left(\hat{P}_{1} \cdot \hat{P}\right)<<1$ because $C_{0} P_{F}$ is approximately the Debye frequency $\omega_{D} \gg T_{c}$ ], and $\epsilon_{1} \sim T$. Thus to lowest order in $\eta$, one may replace $\hat{G}^{R(\text { or } A)}\left(\epsilon_{1}\right)$ by $\pm \hat{\tau}_{z}$, and $\hat{G}^{K}\left(\epsilon_{1}\right)$ by 
$2 \tanh \left(\epsilon_{1} / 2 T\right) \hat{\tau}_{z}$. Equation (5.2) then reduces to

$$
\hat{Z}^{\prime R(\text { or } A)}(\epsilon, t) \simeq\left(-i \epsilon \pm \frac{1}{2 \tau_{E}}\right) \hat{\tau}_{z}-i \hat{\Delta}+i e \phi+\frac{1}{2 \tau_{s}} \hat{\tau}_{z} \hat{G}^{R(\text { or } A)} \hat{\tau}_{z} .
$$

where

$$
\frac{1}{\tau_{E}} \equiv \frac{1}{4} i N(0) g^{2} \int d \Omega_{\hat{P}_{1}} \int \frac{d \epsilon_{1}}{2 \pi}\left[\operatorname{coth}\left(\frac{\epsilon_{1}}{2 T}\right)-\tanh \left(\frac{\epsilon_{1}}{2 T}\right)\right]\left[D_{\hat{P}_{1} \cdot \hat{p}}^{R}\left(\epsilon_{1}\right)-D_{\hat{P}_{1} \cdot \hat{p}}^{A}\left(\epsilon_{1}\right)\right]=\frac{7 \pi}{2} \zeta(3) \frac{N(0) g^{2}}{\left(C_{0} P_{F}\right)^{2}} T^{3} .
$$

While Eqs. (5.5) and (5.6) are only reproductions from the previous work of LO [cf. Ref. 12, Eqs. (16) and (17)], a somewhat detailed justification is presented here to stress the point that the derivation of a set of TDGL presented here is rigorously based on a single small parameter $\eta \equiv|\Delta| / T$.

The further reduction of Eq. (5.1), using Eq. (5.5) and Eq. (4.4) with $\delta \equiv 0$, involves straightforward manipulations of Pauli matrices. [For this purpose, it is convenient to put $\vec{\Delta}=i\left(\operatorname{Re} \Delta \hat{\tau}_{y}+\operatorname{Im} \Delta \hat{\tau}_{x}\right)$. Also, in evaluating the last two terms of Eq. (5.1), we may neglect the ie $\phi$ term in Eq. (5.5), because it would give contributions which are of order $\omega^{2}$.] Since Eq. (5.1) is a $2 \times 2$ matrix equation, it reduces to four scalar equations. From the offdiagonal elements, I obtain (with $\vec{D} \equiv \vec{\nabla}-2 i e \vec{A}$ )

$$
\frac{1}{2} D\left[\alpha \overrightarrow{\mathcal{D}}^{2}(\tilde{\beta}+i \tilde{\gamma})-(\tilde{\beta}+i \tilde{\gamma}) \vec{\nabla}^{2} \alpha\right]^{R(\operatorname{or} A)}=\left(-i \epsilon \pm \frac{1}{2 \tau_{E}}\right)(\tilde{\beta}+i \tilde{\gamma})^{R(\operatorname{or} A)}-\Delta \alpha^{R(\operatorname{or} A)}+\tau_{s}^{-1} \alpha^{R(\operatorname{or} A)}(\tilde{\beta}+i \tilde{\gamma})^{R(\operatorname{or} A)} .
$$

where the + and - signs in \pm are for the retarded $(R)$ and advanced $(A)$ cases, respectively, and

$$
\frac{1}{2} D\left[\alpha \vec{D}^{* 2}(\tilde{\beta}-i \tilde{\gamma})-(\tilde{\beta}-i \tilde{\gamma}) \vec{\nabla}^{2} \alpha\right]^{R(\text { or } A)}=\left(i \epsilon \pm \frac{1}{2 \tau_{E}}\right)(\tilde{\beta}-i \tilde{\gamma})^{R(\operatorname{or} A)}-\Delta^{*} \alpha^{R(\text { or } A)}+\tau_{s}^{-1} \alpha^{R(\operatorname{or} A)}(\tilde{\beta}-i \tilde{\gamma})^{R(\text { or } A)} .
$$

While from the diagonal elements, I obtain

$$
\frac{1}{2} D \vec{\nabla} \cdot\left[(\tilde{\beta}+i \tilde{\gamma}) \vec{D}^{*}(\tilde{\beta}-i \tilde{\gamma})-(\tilde{\beta}-i \tilde{\gamma}) \vec{D}(\tilde{\beta}+i \tilde{\gamma})\right]^{R(\operatorname{or} A)}=\Delta(\tilde{\beta}-i \tilde{\gamma})^{R(\operatorname{or} A)}-\Delta^{*}(\tilde{\beta}+i \tilde{\gamma})^{R(\operatorname{or} A)} .
$$

and (with $\partial_{\epsilon} \equiv \partial / \partial \epsilon, \dot{\Delta} \equiv \partial \Delta / \partial t$ )

$$
\frac{\partial \alpha^{R(\operatorname{or} A)}}{\partial t}=i\left[\dot{\Delta}^{*} \partial_{\epsilon}(\tilde{\beta}+i \tilde{\gamma})+\dot{\Delta} \partial_{\epsilon}(\tilde{\beta}-i \tilde{\gamma})\right]^{R(\text { or } A)}
$$

where used has been made in Eq. (5.9) that $\alpha^{2}+\tilde{\beta}^{2}+\tilde{\gamma}^{2}=1$.

Equation (5.9) may be easily derived from Eqs. (5.7) and (5.8). Less trivially, it is shown in Appendix A that Eq. (5.10) also follows from Eqs. (5.7) and (5.8), if the left-hand-side terms of the latter equations are neglected. This is justifiable because all terms in Eq. (5.10) are already linear in $\omega$. Therefore, to include those left-hand-side terms in that proof would require that Eq. $(5.10)$ be extended to order $D k^{2} \omega$, which is neglected already in Eq. (5.1). Extension of Eq. (5.1) to include this neglected order is not necessary, however, since Eq. (5.10) [and Eq. (5.9)] need not be included in the final set of TDGL equations. On the other hand, Eqs. (5.9) and (5.10) will be used in the subsequent analysis in order to establish the consistency of this final set of equations; but for this purpose, the unextended Eq. (5.10) is already sufficient.

Returning to Eq. (5.7) and (5.8), we see that they imply the following simple relations ${ }^{22}$ :

$$
\alpha^{A}=-\left(\alpha^{R}\right)^{*}, \quad \tilde{\beta}^{A}=\left(\tilde{\beta}^{R}\right)^{*}, \quad \tilde{\gamma}^{A}=\left(\tilde{\gamma}^{R}\right)^{*},
$$

and

$$
(\alpha, \tilde{\beta}, \tilde{\gamma})^{R(\operatorname{or} A)}(-\epsilon, t)=\left(\alpha^{*}, \tilde{\beta}^{*}, \tilde{\gamma}^{*}\right)^{R(\operatorname{or} A)}(\epsilon, t) .
$$

It is therefore only necessary to work with $\alpha^{R}, \tilde{\beta}^{R}$, and $\tilde{\gamma}^{R}$, for which I shall delete the superscript $R$ from now on. They satisfy Eqs. (5.7) and (5.8) with the upper sign in \pm . If Eq. (4.9) is used to transform these equations into the real- $\Delta$ gauge, they would appear as (with $\overrightarrow{\mathrm{Q}} \equiv \vec{\nabla} x-2 e \overrightarrow{\mathrm{A}}$ ):

$$
\begin{aligned}
\frac{1}{2} D\left[\alpha(\vec{\nabla} \pm i \vec{Q})^{2}(\beta \pm i \gamma)-(\beta \pm i \gamma) \vec{\nabla}^{2} \alpha\right] \\
\quad=\left(-i \epsilon+\frac{1}{2} \tau_{E}^{-1}\right)(\beta \pm i \gamma)-|\Delta| \alpha+\tau_{s}^{-1} \alpha(\beta \pm i \gamma)
\end{aligned}
$$


These two equations represent the generalization of Ref. 14, Eq. (5), to the case when Eq. (4.6) should be replaced by Eq. (4.7). While Eq. (5.13) takes the same form as in the "static approximation," I have derived it without making such an approximation, so I have in fact shown that it is also valid when there is a time variation in a scale intrinsic to the GinzburgLandau regime (as opposed to any imposed highfrequency oscillations or rapid temporal variations). The two signs in Eq. (5.13) imply the symmetry relation in Eq. (5.12), so that if one defines

$\alpha=N_{1}+i R_{1}, \quad \beta=N_{2}+i R_{2}, \quad \gamma=N_{3}+i R_{3}$,

then the real parts $N_{1}, N_{2}$, and $N_{3}$ are even functions of $\epsilon$, while the imaginary parts $R_{1}, R_{2}$, and $R_{3}$ are odd. The corresponding definitions in a general gauge are

$\alpha=N_{1}+i R_{1}, \quad \tilde{\beta}=\tilde{N}_{2}+i \tilde{R}_{2}, \quad \tilde{\gamma}=\tilde{N}_{3}+i \tilde{R}_{3}$,

but the even-oddness of the $\tilde{N}$ 's and $\tilde{R}$ 's is the same [cf. Eq. (4.8)].

We can now explore the question of when $\gamma$ may be set equal to zero, as has been done in several previous works. $^{10,14,17}$ Using Eq. (4.13), one may easily derive

$$
\begin{array}{r}
\frac{1}{2} D\left[\alpha\left(\vec{\nabla}^{2}-\overrightarrow{\mathrm{Q}}^{2}\right) \beta-(\alpha / \gamma) \vec{\nabla} \cdot\left(\overrightarrow{\mathrm{Q}} \gamma^{2}\right)-\beta \vec{\nabla}^{2} \alpha\right] \\
=\left(-i \epsilon+\frac{1}{2} \tau_{E}^{-1}\right) \beta-|\Delta| \alpha+\tau_{s}^{-1} \alpha \beta \\
\frac{1}{2} D\left[\alpha\left(\vec{\nabla}^{2}-\overrightarrow{\mathrm{Q}}^{2}\right) \gamma+(\alpha / \beta) \vec{\nabla} \cdot\left(\overrightarrow{\mathrm{Q}} \beta^{2}\right)-\gamma \vec{\nabla}^{2} \alpha\right] \\
=\left(-i \epsilon+\frac{1}{2} \tau_{E}^{-1}\right) \gamma+\tau_{s}^{-1} \alpha \gamma
\end{array}
$$

It appears, therefore, that $\gamma \equiv 0$, if and only if $\vec{\nabla} \cdot\left(\overrightarrow{\mathrm{Q}} \beta^{2}\right)=0$. Since $\beta$ depends also on $\epsilon$, it seems that this condition can be satisfied only if (i) $Q \equiv 0$, (ii) $\vec{Q}$ and $\beta$ are both uniform in space, or (iii) the symmetry of the problem ensures that all spatial gradients are perpendicular to the direction of the local $\vec{Q}$. The condition (iii) is satisfied by many problems of practical importance, including for example, a planar normal-superconducting boundary, and an isolated vortex line, but it is apparently incorrect to assume $\gamma \equiv 0$ for a general situation considered here. On the other hand, remembering that we are only deriving a set of approximate equations based on the smallness of $\Delta / T$, it appears that Eq. (5.17) may allow us to conclude that $\gamma$ is smaller than $\beta$ by at least a factor of $\eta$, so that unless one wishes to study physical effects which depend solely on the nonvanishingness of $\gamma$, otherwise one may indeed set $\gamma=0$ to simplify the set of TDGL equations derived. ${ }^{23}$ So far, I am not aware of any physical phenomena which are indeed caused solely by a small but nonvanishing $\gamma$, but to allow for such possibilities, I shall first complete my derivation of a whole set of TDGL equations without assuming $\gamma \equiv 0$, and then introduce this approximation in Sec. $\mathrm{X}$, in order to arrive at a much simpler set of TDGL equations. Even in this approximation, however, the set of equations derived here still differs in an essential way from the set derived previously, ${ }^{14,17}$ and I believe that whenever the difference is not negligible, it is the set presented in this work that is the more consistent one.

\section{DERIVATION OF THE KINETIC EQUATIONS FOR THE TWO DISTRIBUTION FUNCTIONS}

We now derive two kinetic equations for $\delta f(\epsilon, \overrightarrow{\mathbf{R}}$, ; and $f_{1}(\epsilon, \vec{R}, t)$, using Eqs. (3.21)-(3.23). The "equilibrium distribution function" $f_{0}(\epsilon)$ is equal to $\tanh (\epsilon / 2 T)$ as may be shown by using Eq. (2.1). First, we apply the approximation in Eq. (4.2) to rewrite Eqs. (3.22) and (3.23) as

$$
\begin{aligned}
\hat{G}^{R} & \hat{Y}-\hat{Y} \hat{G}^{A}+\frac{1}{2} i\left\{\hat{G}^{R}, \hat{Y}\right\}_{\epsilon, t}-\frac{1}{2} i\left\{\hat{Y}, \hat{G}^{A}\right\}_{\epsilon, t}=0, \\
\hat{Y}= & \left(\hat{Z}^{\prime R} \hat{f}-\hat{f} \hat{Z}^{\prime A}-\hat{Z}^{\prime K}\right)+\frac{1}{2} i\left\{\hat{Z}^{\prime R}, \hat{f}\right\}_{\epsilon, t}-\frac{1}{2} i\left\{\hat{f} ; \hat{Z}^{\prime A}\right\}_{\epsilon, t}-D \vec{\jmath} \hat{G}^{R} \cdot\left[\vec{\nabla} \delta f+\left(\vec{\nabla} f_{1}-e \dot{\overrightarrow{\mathrm{A}}} f_{0, \epsilon}\right) \hat{\tau}_{z}\right] \\
& -D\left[\vec{\nabla} \delta f+\left(\vec{\nabla} f_{1}-e \dot{\vec{A}} f_{0, \epsilon}\right) \hat{\tau}_{z}\right] \cdot \hat{G}^{A \vec{\partial}},
\end{aligned}
$$

where $f_{0, \epsilon} \equiv \partial f_{0} / \partial \epsilon=(2 T)^{-1} \cosh ^{-2}(\epsilon / 2 T)$, and $\dot{\vec{A}} \equiv \partial \vec{A} / \partial t$. It may be shown that the term " $\left(2 \tau_{s}\right)^{-1} \underline{\tau}_{z} \underline{G}_{\tau_{z}} "$ in $Z^{\prime}$ [cf. Eqs. (3.8) and (3.6a)] gives rise to terms in $Z^{\prime R, A, K}$ which exactly cancel each other in Eq. (3.23), before the approximation in Eq. (4.2) is even employed. ${ }^{24}$ Thus for Eq. (6.2), we may take

$$
\begin{aligned}
& \hat{Z}^{\prime R(\text { or } A)}=\left(-i \epsilon \hat{\tau}_{z}-i \hat{\Delta}+i e \phi\right)+i \hat{\Sigma}^{\mathrm{ph}, R(\text { or } A)}, \\
& Z^{\prime K}=i \hat{\Sigma}^{\mathrm{ph}, K},
\end{aligned}
$$

where all quantities in Eqs. (6.1)-(6.3) are functions of $(\epsilon, \vec{R}, t)$.

In this section, I shall reduce Eqs. (6.1) and (6.2) by using a truncation procedure, which must have also been employed by Kramer and Watts-Tobin, ${ }^{14}$ and also by Schön and Ambegaokar, ${ }^{17}$ because the kinetic equations derived by them are essentially reproduced here, except for some extra improvements, which include the removal of an overrestrictive assumption about $\hat{G}^{R}$ [i.e., Eq. (4.6)], and the avoidance of the additional assumption that a 
"mutilated collision operator" must be used in order to reduce the inelastic collision integrals. It is only after Sec. VIII, where I study the local charge conservation law, which suggets the existence of a problem, I shall then present an argument in Sec. IX, which establishes that the present truncation procedure is actually not a consistent one (according to an order analysis), so that actually more terms must be added to the kinetic equations derived here, in order to make them complete.

The present truncation procedure is based on the intuitive notion that $\delta f$ and $f_{1}$ are both small quantities in comparison with $f_{0}$ (which may be confirmed later with the equations derived), so Eq. (6.1) may be evaluated to its leading order only. This calls for the neglect of the last two terms in Eq. (6.1), and the following evaluation of $Y$.

For the first three terms of $Y$ enclosed in a parentheses, we first note that the $\Sigma^{\text {ph }}$ parts of these terms would cancel out exactly if $f$ is replaced by $f_{0}$, as may be shown with the identify:

$$
\tanh \frac{\epsilon}{2 T}=\frac{\operatorname{coth}\left[\left(\epsilon_{1}-\epsilon\right) / 2 T\right] \tanh \left(\epsilon_{1} / 2 T\right)-1}{\operatorname{coth}\left[\left(\epsilon_{1}-\epsilon\right) / 2 T\right]-\tanh \left(\epsilon_{1} / 2 T\right)} .
$$

The first three terms of $Y$ can then be easily reduced to $^{25}$

$$
\begin{aligned}
2 i\left(\operatorname{Re} \Delta \hat{\tau}_{x}-\operatorname{Im} \Delta \hat{\tau}_{y}\right) & +i\left(\hat{\Sigma}^{\mathrm{ph}, R}-\hat{\Sigma}^{\mathrm{ph}, A}\right)_{\delta f}+i\left[\left(\hat{\Sigma}^{\mathrm{ph}, R}-\hat{\Sigma}^{\mathrm{ph}, A}\right) f_{0}-\hat{\Sigma}^{\mathrm{ph}, \mathrm{K}}\right]_{\hat{f} \rightarrow \delta f}+i\left(\hat{\Sigma}^{\mathrm{ph}, R} \hat{\tau}_{z}-\hat{\tau}_{z} \hat{\Sigma}^{\mathrm{ph}, A}\right) f_{1} \\
& +i\left[\left(\hat{\Sigma}^{\mathrm{ph}, R}-\hat{\Sigma}^{\mathrm{ph}, A}\right) f_{0}-\hat{\Sigma}^{\mathrm{ph}, K}\right]_{\hat{f} \rightarrow f_{1} \hat{\tau}_{z}} .
\end{aligned}
$$

From the next two terms in Eq. (6.2), we keep only

$$
\begin{aligned}
\frac{1}{2} i\left\{-i \epsilon \hat{\tau}_{z}, \delta f+f_{1} \hat{\tau}_{z}\right\}_{\epsilon, t}-\frac{1}{2} i\left\{\delta f+f_{1} \hat{\tau}_{z},-i \epsilon \hat{\tau}_{z}\right\}_{\epsilon, t}+\frac{1}{2} i\left\{-i \hat{\Delta}+i e \phi, f_{0}\right\}_{\epsilon, t}-\frac{1}{2} i\left\{f_{0},-i \hat{\Delta}+i e \phi\right\}_{\epsilon, t} \\
=(\partial / \partial t)\left(\delta f \hat{\tau}_{z}+f_{1}\right)+(-\dot{\hat{\Delta}}+e \dot{\phi}) f_{0, \epsilon} .
\end{aligned}
$$

Finally, for the last two terms in Eq. (6.2), we shall not make any additional approximations. The two kinetic equations for $\delta f$ and $f_{1}$ can then be obtained by taking the trace of Eq. (6.1) (with its last two terms neglected), with and without a factor $\hat{\tau}_{z}$. Simplifications are made using Eqs. (4.4) and (4.5) with $\delta \equiv 0$, and Eq. (5.11) and Eq. (5.15). The results are (after dividing the equation by 4 ):

$$
\begin{aligned}
-D \vec{\nabla} \cdot\left[\left(N_{1}^{2}-\tilde{R}_{1}^{2}-\tilde{R}_{3}^{2}\right) \vec{\nabla} \delta f\right]+D\left(\tilde{N}_{2} \tilde{R}_{3}-\tilde{N}_{3} \tilde{R}_{2}\right) \vec{\nabla} \cdot\left(\vec{\nabla} f_{1}-e \dot{\overrightarrow{\mathrm{A}}} f_{0, \epsilon}\right) \\
-2 D\left[\left(\tilde{R}_{2} \vec{\nabla} \tilde{N}_{3}-\tilde{R}_{3} \vec{\nabla} \tilde{N}_{2}\right)-2 e \overrightarrow{\mathrm{A}}\left(\tilde{N}_{2} \tilde{R}_{2}+\tilde{N}_{3} \tilde{R}_{3}\right)\right] \cdot\left(\vec{\nabla} f_{1}-e \cdot \overrightarrow{\overrightarrow{\mathrm{A}}} f_{0, \epsilon}\right)+\left(\tilde{R}_{2} \operatorname{Re} \dot{\Delta}+\tilde{R}_{3} \operatorname{Im} \dot{\Delta}\right) f_{0, \epsilon} \\
+N_{1} \delta \dot{f}-2\left(\tilde{R}_{3} \operatorname{Re} \Delta-\tilde{R}_{2} \operatorname{Im} \Delta\right) f_{1}=K_{11}[\delta f]+K_{12}\left[f_{1}\right] .
\end{aligned}
$$

and

$$
\begin{array}{r}
-D \vec{\nabla} \cdot\left[\left(N_{1}^{2}+\tilde{N}_{2}^{2}+\tilde{N}_{3}^{2}\right)\left(\vec{\nabla} f_{1}-e \dot{\vec{A}} f_{0, \epsilon}\right)\right]-D\left(\tilde{N}_{2} \tilde{R}_{3}-\tilde{R}_{2} \tilde{N}_{3}\right) \vec{\nabla}^{2} \delta f-2 D\left(\tilde{N}_{2} \vec{\nabla} \tilde{R}_{3}-\tilde{N}_{3} \vec{\nabla} \tilde{R}_{2}\right) \cdot \vec{\nabla} \delta f \\
+4 e D\left(\tilde{N}_{2} \tilde{R}_{2}+\tilde{N}_{3} \tilde{R}_{3}\right) \overrightarrow{\mathrm{A}} \cdot \vec{\nabla} \delta f+\left(\tilde{N}_{3} \operatorname{Re} \dot{\Delta}-\tilde{N}_{2} \operatorname{lm} \dot{\Delta}\right) f_{0, \epsilon}+N_{1}\left(\tilde{f}_{1}+e \dot{\phi} f_{0, \epsilon}\right)+2\left(\tilde{N}_{2} \operatorname{Re} \Delta+\tilde{N}_{3} \operatorname{Im} \Delta\right) f_{1} \\
=K_{21}[\delta f]+K_{22}\left[f_{1}\right] .
\end{array}
$$

where the collision integrals are found to be

$$
\begin{aligned}
& K_{11}[\delta, f] \equiv-\frac{1}{4} i N(0) g^{2} \int d \Omega_{\hat{p}_{1}} \int \frac{d \epsilon_{1}}{2 \pi}\left[D_{\hat{P}_{1} \cdot \hat{p}}^{R}\left(\epsilon_{1}-\epsilon\right)-D_{\hat{p}_{1}, \hat{p}_{1}}\left(\epsilon_{1}-\epsilon\right)\right] \\
& \times\left[N_{1}\left(\epsilon_{1}\right) N_{1}(\epsilon)-\tilde{R}_{2}\left(\epsilon_{1}\right) \tilde{R}_{2}(\epsilon)-\tilde{R}_{3}\left(\epsilon_{1}\right) \tilde{R}_{3}(\epsilon)\right] \\
& \times \frac{\cosh ^{2}(\epsilon / 2 T) \delta f(\epsilon)-\cosh ^{2}\left(\epsilon_{1} / 2 T\right) \delta f\left(\epsilon_{1}\right)}{\sinh \left[\left(\epsilon_{1}-\epsilon\right) / 2 T\right] \cosh \left(\epsilon_{1} / 2 T\right) \cosh (\epsilon / 2 T)} . \\
& K_{12}\left[f_{1}\right] \equiv-\frac{1}{4} i N(0) g^{2} \int d \Omega_{\hat{P}_{1}} \int \frac{d \epsilon_{1}}{2 \pi}\left[D_{\hat{P}_{1} \cdot \hat{p}}^{R}\left(\epsilon_{1}-\epsilon\right)-D_{\hat{P}_{1} \cdot \hat{p}}^{A}\left(\epsilon_{1}-\epsilon\right)\right] \\
& \times\left[\tilde{N}_{2}\left(\epsilon_{1}\right) \tilde{R}_{3}(\epsilon)-\tilde{N}_{3}\left(\epsilon_{1}\right) \tilde{R}_{2}(\epsilon)\right] \\
& \times\left[\frac{\cosh ^{2}(\epsilon / 2 T) f_{1}(\epsilon)-\cosh ^{2}\left(\epsilon_{1} / 2 T\right) f_{1}\left(\epsilon_{1}\right)}{\sinh \left[\left(\epsilon_{1}-\epsilon\right) / 2 T\right] \cosh \left(\epsilon_{1} / 2 T\right) \cosh (\epsilon / 2 T)}-\tanh \left(\frac{\epsilon_{1}}{2 T}\right) f_{1}(\epsilon)\right] .
\end{aligned}
$$




$$
\begin{aligned}
K_{21}[\delta f] \equiv-\frac{1}{4} i N(0) g^{2} \int d \Omega_{\hat{P}_{1}} & \int \frac{d \epsilon_{1}}{2 \pi}\left[D_{\hat{P}_{1}}^{R} \cdot \hat{p}\left(\epsilon_{1}-\epsilon\right)-D_{\hat{P}_{1}, \hat{p}}^{A}\left(\epsilon_{1}-\epsilon\right)\right] \\
& \times\left[\tilde{N}_{2}(\epsilon) \tilde{R}_{3}\left(\epsilon_{1}\right)-\tilde{N}_{3}(\epsilon) \tilde{R}_{2}\left(\epsilon_{1}\right)\right] \frac{\cosh ^{2}(\epsilon / 2 T) \delta f(\epsilon)-\cosh ^{2}\left(\epsilon_{1} / 2 T\right) \delta f\left(\epsilon_{1}\right)}{\sinh \left[\left(\epsilon_{1}-\epsilon\right) / 2 T\right] \cosh \left(\epsilon_{1} / 2 T\right) \cosh (\epsilon / 2 T)},
\end{aligned}
$$

$$
\begin{aligned}
K_{22}\left[f_{1}\right] \equiv-\frac{1}{4} i N(0) g^{2} \int d \Omega_{\hat{P}_{1}} \int \frac{d \epsilon_{1}}{2 \pi}\left[D_{\hat{P}_{1}, \hat{p}}^{R}\left(\epsilon_{1}-\epsilon\right)-D_{\hat{P}_{1} \cdot \hat{p}}^{A}\left(\epsilon_{1}-\epsilon\right)\right] \\
\times\left(\left[N_{1}\left(\epsilon_{1}\right) N_{1}(\epsilon)+\tilde{N}_{2}\left(\epsilon_{1}\right) \tilde{N}_{2}(\epsilon)+\tilde{N}_{3}\left(\epsilon_{1}\right) \tilde{N}_{3}(\epsilon)\right]\right. \\
\times \frac{\cosh ^{2}(\epsilon / 2 T) f_{1}(\epsilon)-\cosh ^{2}\left(\epsilon_{1} / 2 T\right) f_{1}\left(\epsilon_{1}\right)}{\sinh \left[\left(\epsilon_{1}-\epsilon\right) / 2 T\right] \cosh \left(\epsilon_{1} / 2 T\right) \cosh (\epsilon / 2 T)} \\
\left.-\left[\tilde{N}_{2}\left(\epsilon_{1}\right) \tilde{N}_{2}(\epsilon)+\tilde{N}_{3}\left(\epsilon_{1}\right) \tilde{N}_{3}(\epsilon)\right] \tanh \left(\epsilon_{1} / 2 T\right) f_{1}(\epsilon)\right) .
\end{aligned}
$$

For the simpler special case when $\tilde{\beta}+i \tilde{\gamma}=e^{i x} \beta$, and when all but the $N_{1}\left(\epsilon_{1}\right) N_{1}(\epsilon)$ parts in Eqs. (6.8) and (6.11) are neglected, Eckern and Schön ${ }^{15}$ have introduced the concept of "mutilated collision operators" in order to reduce the remaining collision integrals to much simpler forms, namely,

$$
\begin{aligned}
K_{11}+K_{12} \simeq & -\left[N_{1}(\epsilon) / \tau_{E}\right] \delta f \\
K_{21}+K_{22}= & -\left[N_{1}(\epsilon) / \tau_{E}\right] f_{1} \\
& +\left[N_{1}(\epsilon) f_{0, \epsilon}(\epsilon) / 2 \tau_{E}\right] \\
& \times \int_{-\infty}^{\infty} N_{1}\left(\epsilon_{1}\right) f_{1}\left(\epsilon_{1}\right) d \epsilon_{1}
\end{aligned}
$$

[Strictly speaking, the second term in Eq. (6.13) should be further divided by $\frac{1}{2} \int_{-\infty}^{\infty} N_{1}\left(\epsilon_{1}\right) f_{0, \epsilon}\left(\epsilon_{1}\right) d \epsilon_{1}$, but this quantity differs from unity only to order $\eta^{2}$. Also, the $\epsilon$ dependence of $\tau_{E}^{-1}$ is neglected for the same reason.] The approximate forms in Eqs. (6.12) and (6.13) are used by Kramer and Watts-Tobin ${ }^{14}$ and by Schön and Ambegaokar ${ }^{17}$ in order to simplify their kinetic equations. The former two authors have further reduced Eq. (6.13) to the form:

$$
K_{21}+K_{22}=-\left[N_{1}(\epsilon) / \tau_{E}\right]\left(f_{1}+e \phi f_{0, \epsilon}\right),
$$

by employing an approximate charge neutrality condition, i.e., $\rho \simeq 0$, which may be verified selfconsistently.

I find that Eq. (6.12), and a slight generalization of Eq. (6.14), viz.,

$$
K_{21}+K_{22} \simeq-\left[N_{1}(\epsilon) / \tau_{E}\right]\left(f_{1}+e \phi f_{0, \epsilon}\right)+\left[N_{1}(\epsilon) f_{0, \epsilon} / 2 \tau_{E}\right] \int_{-\infty}^{\infty} N_{1}\left(\epsilon_{1}\right)\left[f_{1}\left(\epsilon_{1}\right)+e \phi f_{0, \epsilon_{1}}\left(\epsilon_{1}\right)\right] d \epsilon_{1} .
$$

(which gives a vanishing integral of $\epsilon$ ) can actually be rigorously justified by invoking the sole small parameter $|\Delta| / T$. This means that the so called

"mutilated-collision-operator" approximation is actually not an additional assumption in the derivation of a consistent set of TDGL equations. The actual de- tailed justification of Eqs. (6.12) and (6.15) has been presented in Appendix B, since only its conclusions are needed for the rest of this paper.

If we use Eq. (4.9) to transform Eqs. (6.6) and (6.7) into the real- $\Delta$ gauge, the latter equations would become

$$
\begin{aligned}
& -D \vec{\nabla} \cdot\left[\left(N_{1}^{2}-R_{2}^{2}-R_{3}^{2}\right) \vec{\nabla} \delta f\right]+D\left(N_{2} R_{3}-N_{3} R_{2}\right) \vec{\nabla} \cdot\left(\vec{\nabla} f_{1}-e \dot{\overrightarrow{\mathrm{A}}} f_{0, \epsilon}\right) \\
& -2 D\left[\left(R_{2} \vec{\nabla} N_{3}-R_{3} \vec{\nabla} N_{2}\right)+\overrightarrow{\mathrm{Q}}\left(N_{2} R_{2}+N_{3} R_{3}\right)\right] \cdot\left(\vec{\nabla} f_{1}-e \dot{\overrightarrow{\mathrm{A}}} f_{0, \epsilon}\right)+R_{2}|\dot{\Delta}| f_{0, \epsilon} \\
& +N_{1}\left(\partial_{1}+\tau_{E}^{-1}\right) \delta f \\
& +R_{3}|\Delta|\left(2 f_{1}-\dot{\chi} f_{0, \epsilon}\right)=0
\end{aligned}
$$

and

$$
\begin{gathered}
-D \vec{\nabla} \cdot\left[\left(N_{1}^{2}+N_{2}^{2}+N_{3}^{2}\right)\left(\vec{\nabla} f_{1}-e \dot{\overrightarrow{\mathrm{A}}} f_{0, \epsilon}\right)\right]-D\left(N_{2} R_{3}-N_{3} R_{2}\right) \vec{\nabla}^{2} \delta f-2 D\left[\left(N_{2} \vec{\nabla} R_{3}-N_{3} \vec{\nabla} R_{2}\right)+\overrightarrow{\mathrm{Q}}\left(N_{2} R_{2}+N_{3} R_{3}\right)\right] \\
\cdot \vec{\nabla} \delta f+N_{3}|\dot{\Delta}| f_{0, \epsilon}+N_{1}\left(\partial_{t}+\tau_{E^{-1}}^{-1}\right)\left(f_{1}+e \phi f_{0, \epsilon}\right)+N_{2}|\Delta|\left(2 f_{1}-\dot{\chi} f_{0, \epsilon}\right) \\
=\tau_{E}^{-1} f_{0, \epsilon} \int_{0}^{\infty} N_{1}\left(\epsilon^{\prime}\right)\left[f_{1}\left(\epsilon^{\prime}\right)+e \phi f f_{0, \epsilon^{\prime}}\left(\epsilon^{\prime}\right)\right] d \epsilon^{\prime}
\end{gathered}
$$


These equations are manifestly gauge invariant, if one assumes that $\delta f$ and $f_{1}+e \phi f_{0, \epsilon}$ are gauge invariant quantities. When approximate charge neutrality $\rho=0$ is further taken into consideration, the righthand side of Eq. (6.17) would be approximated by zero [cf. Eq. (7.12)], and an integral-differential equation would reduce simply to a differential equation, which would be very convenient for practical applications. However, I shall not invoke such an approximation at the stage of deriving a general set of TDGL equations, since I wish to examine such a set of equations against the satisfaction of a local charge conservation law. (This approximation will be considered in Sec. X.)

To conclude this section, we note that Eq. (6.16), and Eq. (6.17) without the right-hand side, would reduce to Eqs. (6) and (7) of Ref. 14, if $N_{3}$ and $R_{3}$ are set equal to zero. This corresponds to assuming Eq. (4.6), which, as I have already pointed out in Sec. $\mathbf{V}$, is not exactly valid in a general magnetic field. Equations (6.16) and (6.17), therefore, represent the appropriate extensions to the more general cases, when Eq. (4.6) should be replaced by Eq. (4.7). Finally, I wish to remind the readers once again that, although the truncation procedure used in this sec- tion is capable of reproducing earlier results and even extending them, I shall demonstrate in Secs. VIII and IX that this truncation procedure is actually not selfconsistent, and Eq. (6.16) and (6.17) are actually incomplete.

\section{DERIVATION OF EQUATIONS FOR THE ORDER PARAMETER, AND FOR THE CURRENT AND CHARGE DENSITIES}

For the order parameter equation, we may start with

$$
\begin{aligned}
\Delta & =|\lambda|\left\langle\psi_{1}(x) \psi_{l}(x)\right\rangle \\
& =\frac{1}{2} \pi N(0)|\lambda| \int \frac{d \epsilon}{2 \pi}\left[\hat{G}^{K}(\epsilon, \overrightarrow{\mathrm{R}}, t)\right]_{12} \\
& =\frac{1}{8} N(0)|\lambda| \int d \epsilon \operatorname{Tr}\left[\left(\hat{\tau}_{x}-i \hat{\tau}_{y}\right)\left(\hat{G}^{R} \circ \hat{f}-\hat{f} \circ \hat{G}^{A}\right)\right],
\end{aligned}
$$

where $\lambda$ is the BCS coupling constant of a weakcoupling superconductor. Using the approximation in Eq. (4.2), Eq. (7.1) is first reduced to

$$
\begin{aligned}
& {[N(0)|\lambda|]^{-1} \Delta=\frac{1}{8} \int d \epsilon\left(\left(f_{0}+\delta f\right) \operatorname{Tr}\left[\left(\hat{\tau}_{x}-i \hat{\tau}_{y}\right)\left(\hat{G}^{R}-\hat{G}^{A}\right)\right]-f_{1} \operatorname{Tr}\left[\left(\hat{\tau}_{x}-i \hat{\tau}_{y}\right)\left(\hat{G}^{R}+\hat{G}^{A}\right)\right]\right.} \\
&\left.-\frac{1}{2} i\left\{f_{0}+\delta f, \operatorname{Tr}\left[\left(\hat{\tau}_{x}-i \hat{\tau}_{y}\right)\left(\hat{G}^{R}-\hat{G}^{A}\right)\right]\right\}_{\epsilon, t}+\frac{1}{2} i\left\{f_{1}, \operatorname{Tr}\left[\left(\hat{\tau}_{x}-i \hat{\tau}_{y}\right)\left(\hat{G}^{R}+\hat{G}^{A}\right)\right]\right\}_{\epsilon, t}\right)
\end{aligned}
$$

In this section, I shall again aim at reproducing the results of Kramer and Watts-Tobin, ${ }^{14}$ and of Schön and Ambegaokar, ${ }^{17}$ with only the slight generalization of allowing $\gamma$ and $\tau_{s}^{-1}$ not equal to zero. For this purpose, I shall neglect the $\delta f$ and $f_{1}$ terms located near the end of Eq. (7.2), based on the simple reasoning that $\delta f$ and $f_{1}$ are small in comparison with $f_{0}$, which actually can be verified by using Eqs. (6.16) and (6.17). In Secs. VIII and IX, I shall uncover a subtle point in the order analysis, which leads to new terms to be added into Eqs. (6.16) and (6.17).
In discussing this point, I shall also return to the omitted $\delta f$ and $f_{1}$ terms in Eq. (7.2).

The evaluation of the remaining terms in Eq. (7.2) is actually straightforward, so I shall only remark that for the $f_{0}$ parts of the first and the third terms, the energy integration may be performed by deforming the integration contour into the complex- $\epsilon$ plane, always to encircle only the poles of $\tanh (\epsilon / 2 T)$ at $\epsilon=i(2 n+1) \pi T>>|\Delta|$, so for $\hat{G}^{R}$ and $\hat{G}^{A}$ one may use their high-energy expansions, obtainable easily from Eqs. (5.7) and (5.8):

$$
\tilde{\beta} \pm i \tilde{\gamma}=\frac{\Delta\left(\text { or } \Delta^{*}\right)}{-i \epsilon+\tau_{s}^{-1}+\frac{1}{2} \tau_{E}^{-1}}+\frac{1}{2}\left(\frac{D \vec{D}^{2} \Delta\left(\text { or } \vec{D}^{* 2} \Delta^{*}\right)}{\left(-i \epsilon+\tau_{s}^{-1}+\frac{1}{2} \tau_{E}^{-1}\right)^{2}}+\frac{\left(-i \epsilon+\frac{1}{2} \tau_{E}^{-1}\right)|\Delta|^{2} \Delta\left(\text { or } \Delta^{*}\right)}{\left(-i \epsilon+\tau_{s}^{-1}+\frac{1}{2} \tau_{E}^{-1}\right)^{4}}\right)
$$

Eq. (6.2) can then be reduced to

$$
\bar{A} \Delta-\bar{B}|\Delta|^{2} \Delta-C\left(\dot{\Delta}-D \bar{D}^{2} \Delta\right)+\int_{0}^{\infty} d \epsilon\left[\delta f\left(\tilde{R}_{2}+i \tilde{R}_{3}\right)+i f_{1}\left(\tilde{N}_{2}+i \tilde{N}_{3}\right)\right]=0
$$

where use has been made that the integrand is an even function of $\epsilon$. [I.e., $\delta f$ is odd, $f_{1}$ is even, as may be shown with Eqs. (6.16) and (6.17). The rest follows from the discussion below Eq. (5.14).] The coefficients $\bar{A}$, $\bar{B}$, and $C$ are essentially those defined previously by the author, ${ }^{9}$ except that they are slightly generalized to in- 
clude the depairing effects of the phonon field:

$$
\bar{A} \equiv \ln \left(T_{c} / T\right)+\psi\left(\frac{1}{2}+\rho_{t} T / T_{c}\right)-\psi\left(\frac{1}{2}+\rho_{t}\right),
$$

$\bar{B} \equiv-(4 \pi T)^{-2}\left[\psi^{(2)}\left(\frac{1}{2}+\rho_{t}\right)+\frac{1}{3} \rho_{s} \psi^{(3)}\left(\frac{1}{2}+\rho_{t}\right)\right]$,

and

$C \equiv(4 \pi T)^{-1} \psi^{(1)}\left(\frac{1}{2}+\rho_{t}\right)$, where

$\rho_{t} \equiv \rho_{s}+\rho_{E}, \quad \rho_{s} \equiv\left(2 \pi \tau_{s} T\right)^{-1}, \quad \rho_{E} \equiv\left(4 \pi \tau_{E} T\right)^{-1}$,

and $T_{c}$ is defined by

$$
\ln \left(T_{c 0} / T_{c}\right)=\psi\left(\frac{1}{2}+\rho_{t} T / T_{c}\right)-\psi\left(\frac{1}{2}\right),
$$

with $T_{c 0}$ being the transition temperature in the absence of all pair-breaking effects.

If Eq. (4.9) is used to transform Eq. (7.4) into the real- $\Delta$ gauge, it then reads

$$
\bar{A} \Delta-\bar{B}|\Delta|^{2} \Delta-C\left(\dot{\Delta}-D \vec{D}^{2} \Delta\right)+(\Delta /|\Delta|) \int_{0}^{\infty} d \epsilon\left[\delta f\left(R_{2}+i R_{3}\right)+i f_{1}\left(N_{2}+i N_{3}\right)\right]=0
$$

This equation, with $N_{3}, R_{3}$, and $\rho_{s}$ set equal to zero, and with all the effects of $\rho_{E}$ in Eqs. (7.5a) - (7.5e) neglected, reduces to Eq. (1) of Ref. 14, or Eqs. (25) and (26) of Ref. 17. So Eq. (7.6) apparently represents the appropriate extension to the cases of nonvanishing $\gamma$ and/or $\tau_{s}^{-1}$. However, this equation remains unsatisfactory, because it is only approximately invariant under the gauge transformation $\phi \rightarrow \phi-\Lambda, \overrightarrow{\mathrm{A}} \rightarrow \overrightarrow{\mathrm{A}}+\vec{\nabla} \Lambda, \Delta \rightarrow \Delta \exp (2 i e \Lambda)$, and $f_{1} \rightarrow f_{1}+e \dot{\Lambda} f_{0, \epsilon}$, mainly because that

$\int_{0}^{\infty} d \epsilon\left(N_{2}+i N_{3}\right) f_{0, \epsilon}$ is only approximately equal to
$2 C|\Delta|$. [I.e., with an error of order $\eta^{2}$ smaller than the dominant part, as may be shown using Eq. (7.3).] While the TDGL equations are supposed to be valid only approximately, it is still desirable for these equations to obey an exact gauge invariance, because otherwise, the same physical quantity calculated by applying these equations in two different gauges would not be guaranteed to agree. As a matter of fact, such two results can in principle differ by a very large amount, because a nongauge-invariant quantity can be made to take any value by choosing a suitable gauge. We therefore propose that Eq. (7.6) should be changed to

$$
\bar{A} \Delta-\bar{B}|\Delta|^{2} \Delta-C\left(\dot{\Delta}+2 i e \phi \Delta-D \vec{D}^{2} \Delta\right)+(\Delta /|\Delta|) \int_{0}^{\infty} d \epsilon\left[\delta f\left(R_{2}+i R_{3}\right)+i\left(f_{1}+e \phi f_{0, \epsilon}\right)\left(N_{2}+i N_{3}\right)\right]=0 .
$$

which may also be written as

$$
\begin{aligned}
\bar{A}|\Delta|-\bar{B}|\Delta|^{3}-C[|\dot{\Delta}|+i|\Delta|(\dot{\chi}+2 e \phi)-D(\vec{\nabla} & \left.+i \overrightarrow{\mathrm{Q}})^{2}|\Delta|\right] \\
& +\int_{0}^{\infty} d \epsilon\left[\delta f\left(R_{2}+i R_{3}\right)+i\left(f_{1}+e \phi, f_{0, \epsilon}\right)\left(N_{2}+i N_{3}\right)\right]=0,
\end{aligned}
$$

which demonstrates that the equation is now exactly gauge invariant.

The current and charge densities may be derived by starting with the following basic equations ${ }^{11,12}$ :

$$
\begin{aligned}
\rho & =-N(0) e\left(2 e \phi+\frac{1}{4} \int_{-\infty}^{\infty} d \epsilon \operatorname{Tr} \hat{G}^{K}\right), \\
\overrightarrow{\mathrm{j}} & =-\left(\frac{1}{4}\right) N(0) e \int_{-\infty}^{\infty} d \epsilon \int\left(d \Omega_{\hat{\rho}} / 4 \pi\right)(\overrightarrow{\mathrm{p}} / m) \operatorname{Tr}\left(\hat{\tau}_{z} \hat{G}_{\hat{p}}^{K}\right) \\
& =\left(\frac{1}{4}\right) N(0) e D \int_{-\infty}^{\infty} d \epsilon \operatorname{Tr}\left[\hat{\tau}_{z}\left(\hat{G}^{R} \circ \vec{\partial} \circ \hat{G}^{K}+\hat{G}^{K} \circ \vec{\partial} \circ \hat{G}^{A}\right)\right] .
\end{aligned}
$$

Using $\hat{G}^{K}=\hat{G}^{R} \circ \hat{f}-\hat{f} \circ \hat{G}^{A}$, and Eq. (4.2) with its second term neglected, one may easily evaluate $\rho$ to be

$$
\rho=-2 e N(0)\left(e \phi+\int_{0}^{\infty} d \epsilon N_{1} f_{1}\right)
$$

While this equation is exactly the same as one obtained by Kramer and Watts-Tobin [Ref. 14, Eq. (3)], and by Schön and Ambegaokar [Ref. 17, Eq. (31)], it remains not completely satisfactory, because it is only approximately gauge invariant. This is because $\int_{0}^{\infty} d \epsilon N_{1} f_{0, \epsilon}$ is only approximately equal to unity, with an error of the order $\eta^{2}$. This problem arises because the first term in Eq. (7.11) is an exact result, while the second term is evaluated only approximately. To regain exact gauge invariance, therefore, I propose to deliberately introduce a small error in the first term, so it will read

$$
\rho=-2 e N(0) \int_{0}^{\infty} d \epsilon N_{1}\left(f_{1}+e \phi f_{0, \epsilon}\right) .
$$


I emphasize that it would be wrong to change $N_{1}$ to unity in the second term of Eq. (7.11), because the error so introduced would not be small.

For the current density, we need to keep the second term of Eq. (4.2) in $\left[\hat{\partial}, f_{0}\right]^{\circ}=-e \dot{A} f_{0, \epsilon}$ only:

$$
\overrightarrow{\mathrm{j}}=\left(\frac{1}{4}\right) e N(0) D \int_{-\infty}^{\infty} d \epsilon \operatorname{Tr}\left\{\tau_{z}\left[\left(\hat{G}^{R} \vec{\partial} \hat{G}^{R}-\hat{G}^{A} \vec{\partial} \hat{G}^{A}\right)\left(f_{0}+\delta f+f_{1} \hat{\tau}_{z}\right)\right]-\hat{G}^{R}\left[\vec{\nabla} \delta f+\left(\vec{\nabla} f_{1}-e \dot{\overrightarrow{\mathrm{A}}} f_{0, \epsilon}\right) \hat{\tau}_{z}\right] \hat{G}^{A}\right\}
$$

Further evaluation of the traces and the energy integral of the $f_{0}$ term gives

$$
\begin{aligned}
\overrightarrow{\mathrm{j}}= & -2 \operatorname{ieN}(0) D C\left(\Delta^{*} \overrightarrow{\mathrm{D}} \Delta-\Delta \vec{D}^{*} \Delta^{*}\right) \\
& +2 e N(0) D \int_{0}^{\infty} d \epsilon\left\{\left[\left(\tilde{N}_{2} \vec{\nabla} \tilde{R}_{3}+\tilde{R}_{2} \vec{\nabla} \tilde{N}_{3}-\tilde{N}_{3} \vec{\nabla} \tilde{R}_{2}-\tilde{R}_{3} \vec{\nabla} \tilde{N}_{2}\right)-4 e \overrightarrow{\mathrm{A}}\left(\tilde{N}_{2} \tilde{R}_{2}+\tilde{N}_{3} \tilde{R}_{3}\right)\right] \delta f\right. \\
& \left.\quad+\left(\tilde{N}_{2} \tilde{R}_{3}-\tilde{R}_{2} \tilde{N}_{3}\right) \vec{\nabla} \delta f+\left(N_{1}^{2}+\tilde{N}_{2}^{2}+\tilde{N}_{3}^{2}\right)\left(\vec{\nabla} f_{1}-e \dot{\overrightarrow{\mathrm{A}}} f_{0, \epsilon}\right)\right\} .
\end{aligned}
$$

This equation may be easily transformed into the real- $\Delta$ gauge, giving:

$$
\begin{aligned}
\overrightarrow{\mathrm{j}}=4 e N(0) C D|\Delta|^{2} \overrightarrow{\mathrm{Q}}+2 e N(0) D \int_{0}^{\infty} d \epsilon\{[ & \left.\left(N_{2} \vec{\nabla} R_{3}+R_{2} \vec{\nabla} N_{3}-N_{3} \vec{\nabla} R_{2}-R_{3} \vec{\nabla} N_{2}\right)+2 \overrightarrow{\mathrm{Q}}\left(N_{2} R_{2}+N_{3} R_{3}\right)\right] \delta f \\
& \left.+\left(N_{2} R_{3}-R_{2} N_{3}\right) \vec{\nabla} \delta f+\left(N_{1}^{2}+N_{2}^{2}+N_{3}^{2}\right)\left(\vec{\nabla} f_{1}-e \dot{\overrightarrow{\mathrm{A}}} f_{0, \mathrm{\epsilon}}\right)\right\}
\end{aligned}
$$

which is manifestly gauge invariant. If $N_{3}, R_{3}$, and $\rho_{s}$, and $\rho_{E}$ are set equal to zero, and the following expression is used for the normal state conductivity $\sigma_{0}$ :

$$
\sigma_{0}=2 e^{2} N(0) D,
$$

then Eq. (7.15) reduces to Eq. (2) of Ref. 14, or Eqs. (28) and (29) of Ref. 17, showing that we have obtained the desired extension of $\vec{j}$ for this section.

\section{CHECKING LOCAL CONSERVATION OF CHARGE}

In Secs. IV-VII, I have derived a set of TDGL equations which differs from a set derived previously only in the following improvements: (i) Equation (4.6) has been replaced by a more generally valid Eq. (4.7) for representing $\hat{G}^{R}$ (or $A$ ). (ii) The derivation of equations for the spectral functions has been extended beyond the static approximation employed previously. ${ }^{14}$ (iii) It is shown that the so-called "mutilated-collision-operator" approximation introduced by Eckern and Schön ${ }^{15}$ is actually a direct consequence of the smallness of $|\Delta| / T$, and so it is not an additional approximation. (iv) Small changes are made to the equations derived in order to convert an approximation gauge invariance to an exact gauge invariance, which I believe is very desirable. In an arbitrary gauge, the resulting set of equations is the collection of Eqs. (5.7) and (5.8) (with the upper signs and with the superscripts deleted); Eqs. (6.5) and (6.6) [with the collision integrals approximated by Eqs. (6.12) and (6.15)]; plus Eqs. (7.8), (7.12), and (7.14). On the other hand, in the real- $\Delta$ gauge, the set of equations would be represented by Eq. (5.13); Eqs. (6.16) and (6.17); and Eqs. (7.7) [or (7.8)], (7.12) and (7.15).

The above set of equations has already passed an important test, namely, gauge invariance. I now wish to subject it to another important test, i.e., local con- servation of charge, or $\dot{\rho}+\vec{\nabla} \cdot \vec{j}=0$. If the derivation of a set of approximate equations involves keeping all terms above a predetermined order, and neglecting all terms of higher order in smallness, then local charge conservation law should be satisfied exactly. In practice, it is not necessary, nor convenient, to ensure that all negligibly small quantities are removed from the equations derived. For the present problem of deriving a set of TDGL equations, which involves an energy variable $\epsilon$ that is integrated in some equations, and not in some others, it is in fact impossible to achieve the goal of removing all negligibly small contributions. (See Sec. IX for further elaboration of this point.) Thus we expect $\dot{\rho}+\vec{\nabla} \cdot \vec{j}=0$ to be satisfied only approximately. Approximate satisfaction of this condition, however, is not as undesirable as an approximate satisfaction of a gauge invariance, since the error in the former can not be made arbitrarily large, simply by a change of gauge, but in fact will always remain as being negligibly small.

Before we check the approximate satisfaction of $\dot{\rho}+\nabla \cdot \mathrm{j}=0$, we must first determine the order of the leading contributions to this equation. This may be seen from the current expression, Eq. (7.15), which is made of a static term $4 e N(0) C D|\Delta|^{2} \overrightarrow{\mathrm{Q}}$, generally identified as the supercurrent $\overrightarrow{\mathrm{j}}_{s}$, and the remaining dynamic contributions, which may be identified as the normal current $\vec{j}_{n}$. Expecting that the leading order of $\vec{j}_{n}$ should be no larger than that of 
$j_{s}$, we conclude that the dominant contribution for $\vec{j}$ is of order $e N(0) v_{F} \eta^{2} \Delta$. Then in the equation $\dot{\rho}+\vec{\nabla} \cdot \vec{j}=0$, the largest contributions must be

$$
\begin{aligned}
& \rho \sim e N(0) \eta|\Delta|, \quad \vec{j} \sim e N(0) v_{F} \eta^{2}|\Delta|, \\
& \dot{\rho} \sim \vec{\nabla} \cdot \vec{j} \sim e N(0) \eta^{2}|\Delta|^{2},
\end{aligned}
$$

which suggest that if we find $\dot{\rho}+\vec{\nabla} \cdot \vec{j}<<O\left(e N(0) \eta^{2}|\Delta|^{2}\right)$, then the local charge conservation law is approximately satisfied.

I shall now evaluate $\dot{\rho}+\vec{\nabla} \cdot \vec{j}$ in the gauge in which $\Delta$ is real. Using Eqs. (7.12) and (7.15), and with a straightforward substitution of Eq. (6.17), I first obtain

$$
\begin{aligned}
v=\frac{\dot{\rho}+\vec{\nabla} \cdot \overrightarrow{\mathrm{j}}}{2 e N(0)}= & -\int_{0}^{\infty} d \epsilon \dot{N}_{1}\left(f_{1}+e \phi f_{0, \epsilon}\right)+2 C D \vec{\nabla} \cdot\left(|\Delta|^{2} \overrightarrow{\mathrm{Q}}\right) \\
& +D \int_{0}^{\infty} d \epsilon \delta f \vec{\nabla} \cdot\left[\left(N_{2} \vec{\nabla} R_{3}+R_{2} \vec{\nabla} N_{3}-N_{3} \vec{\nabla} R_{2}-R_{3} \vec{\nabla} N_{2}\right)+2 \overrightarrow{\mathrm{Q}}\left(N_{2} R_{2}+N_{3} R_{3}\right)\right] \\
& +\int_{0}^{\infty} d \epsilon\left[N_{3}|\dot{\Delta}| f_{0, \epsilon}+N_{2}|\Delta|\left(2 f_{1}-\dot{\chi} f_{0, \epsilon}\right)\right]
\end{aligned}
$$

Now using Eq. (5.9) transformed into the real- $\Delta$ gauge, which is the exact consequence of Eq. (5.13), we may derive

$$
\begin{aligned}
D \vec{\nabla} \cdot\left[\left(N_{2} \vec{\nabla} R_{3}+R_{2}\right.\right. & \left.\vec{\nabla} N_{3}-N_{3} \vec{\nabla} R_{2}-R_{3} \vec{\nabla} N_{2}\right) \\
& \left.+2 \overrightarrow{\mathrm{Q}}\left(N_{2} R_{2}+N_{3} R_{3}\right)\right]=2 R_{3}|\Delta| .
\end{aligned}
$$

Taking the imaginary part of Eq. (7.8), we may also derive:

$$
\begin{aligned}
C D \nabla \cdot\left(|\Delta|^{2} Q\right)= & C|\Delta|^{2}(\dot{\chi}+2 e \phi) \\
-\int_{0}^{\infty} d \epsilon & {\left[R_{3}|\Delta| \delta f+N_{2}|\Delta|\right.} \\
& \left.\times\left(f_{1}+e \phi f_{0, \epsilon}\right)\right] .
\end{aligned}
$$

Substituting these two relations into Eq. (8.1), we obtain the following simple result for the quantity $U$ which measures the extent of violation of the local charge conservation law:

$$
\begin{aligned}
v= & -\int_{0}^{\infty} d \epsilon \dot{N}_{1}\left(f_{1}+e \phi f_{0, \epsilon}\right)+|\dot{\Delta}| \int_{0}^{\infty} N_{3, f_{0, \epsilon} d \epsilon} \\
& +|\Delta|(\dot{x}+2 e \phi)\left(2 C|\Delta|-\int_{0}^{\infty} N_{2} f_{0, \epsilon} d \epsilon\right) .
\end{aligned}
$$

Using the high-energy expansion given in Eq. (7.3), one may show that for $\epsilon \sim T$,

$$
\begin{aligned}
& N_{3}=0+O\left(\eta^{3}\right), \\
& N_{2} \simeq \frac{\left(\tau_{s}^{-1}+\frac{1}{2} \tau_{E}^{-1}\right)|\Delta|}{\epsilon^{2}+\left(\tau_{s}^{-1}+\frac{1}{2} \tau_{E}^{-1}\right)^{2}}+O\left(\eta^{3}\right),
\end{aligned}
$$

which implies that the last two terms of Eq. (8.4) are of order $\eta^{4}|\Delta|^{2}$, and are therefore negligible. The first term in Eq. (8.4) will be discussed in the next section, since it leads to the discovery of new terms for the kinetic equations.

\section{NEW CONTRIBUTIONS TO THE TDGL EQUATIONS DERIVED}

In the last section, I have shown that the set of TDGL equations derived so far implies

$\dot{\rho}+\vec{\nabla} \cdot \overrightarrow{\mathrm{j}}=-2 e N(0) \int_{0}^{\infty} d \epsilon \dot{N}_{1}\left(f_{1}+e \phi f_{0, \epsilon}\right)$.

There are two possible conclusions about this remaining apparent violation of the local charge conservation law: (i) The quantity that is on the right-hand side of Eq. (9.1) is much smaller than the nonnegligible order $e N(0) \eta^{2}|\Delta|^{2}$, and may therefore be ignored. (ii) The set of TDGL equations derived so far is incomplete. There are important new contributions to the kinetic equations derived which can lead to a cancellation of this quantity. Odd as it might seem, I shall show below that both of these "possible conclusions" are actually true at the same time. First of all, an actual estimation of the right-hand side of Eq. (9.1) [using Eq. (6.17)] indicates that this quantity is probably no larger than the order eN $(0) \eta^{3}|\Delta|^{2} .^{26}$ Thus the equation $\dot{\rho}+\vec{\nabla} \cdot \vec{j}=0$ is at least satisfied in its leading order $e N(0) \eta^{2}|\Delta|^{2}$, and the "possible conclusion (i)" is in a sense true. However, since an exact theory would satisfy $\dot{\rho}+\vec{\nabla} \cdot \vec{j}=0$ exactly, there must be some contributions to this set of dynamic equations that are neglected so far, which can lead to a cancellation of this undesired term in Eq. (9.1). The important question is whether those contributions are all negligible in their respective equations. However against intuition it might seem to be, the answer is that they are not all negligible (mainly in the kinetic equations for $\delta f$ and $f_{1}$ ), so that the "possible conclusion (ii)" as given above is also true.

The key to this apparent enigma lies in a subtle point in the order analysis, which is caused by the existence of two important energy scales $(|\Delta|$ and $T)$, for a superconductor with a finite-energy gap. For 
comparison, I mention my earlier study of a set of TDGL equations for a gapless superconductor ${ }^{9}$ (due in its earlier form to Gor'kov and Éliashberg ${ }^{7,8}$ ), where all important physics occurs in the energy scale $\epsilon>|\Delta|$, so one may use $\epsilon \sim T\left(\sim \tau_{s}^{-1}\right)$ in all order analyses for a gapless superconductor. Now for superconductors with a finite gap, the singular nature of the spectral functions at the gap frequency implies that the contribution from the energy range $\epsilon \leq|\Delta|$ of an $\epsilon$ integral can be comparable to, or even larger than, the contribution from the energy range $\epsilon \sim T$. One must therefore consider both $\epsilon \sim T$ and $\epsilon \sim|\Delta|$ in an order analysis, unless one is sure that only one scale is involved. In particular, the matrix distribution function $\hat{f}$ has been put as $\left(f_{0}+\delta f\right) \hat{1}+f_{1} \hat{\tau}_{2}$, with $\delta f$ and $f_{1}$ assumed small, which has formed the basis of our truncation procedure used so far. Now if we assume that $\delta f$ and $f_{1}$ are only as small as being of order $\eta$, then because $f_{0}$ varies only in the scale $T$, while for $\epsilon \leq|\Delta|, \delta f$ and $f_{1}$ obviously also vary in the scale $|\Delta|$, so $\partial\left(\delta f^{\prime}\right) / \partial \epsilon$ and $\partial f_{1} / \partial \epsilon$ must be treated as quantities of the same order as $\partial f_{0} / \partial \epsilon$. A conceptual complication arises however, because Eqs. (6.16) and (6.17) indicate that only $\delta f$ is of order $\eta$ in the energy range $\epsilon \leq|\Delta|$ (for $\tau_{E}^{-1} \leq \eta|\Delta|$ only, a $\tau_{E}^{-1} \geq|\Delta|$ would make $\delta f$ further smaller by a factor $\eta$, which would allow further simplification of the TDGL equations derived), while $f_{1}+e \phi f_{0, \epsilon}$ is actually only of order $\eta^{2}$ for all $\epsilon \leq T .^{26}$ The existence of this "additional smallness" means that we can make further approximations later, but for the moment I shall simply assume $\delta f \sim f_{1} \sim \eta$. So far I have pointed out that energy differentiation can change the relative order of two quantities. There is also the point that energy integration can cause such a change of order as well. Consider two functions of energy $p(\epsilon)$ and $q(\epsilon)$, which are comparable in magnitude for $\epsilon \leq|\Delta|$. If $p(\epsilon)$ is large only for $\epsilon \leq|\Delta|$, while $q(\epsilon)$ stays large for all $\epsilon \leq T$, then clearly $\int_{0}^{\infty} P(\epsilon) d \epsilon$ will be smaller than $\int_{0}^{\infty} q(\epsilon) d \epsilon$ by a factor $\eta$. In the following, when we look for new contributions to the TDGL equations, we shall ignore this last point about "change of order by integration," so that we will obtain an "undersimplified" set of equations. This is so that local conservation of charge, which is an energy-integrated relation, can be more easily verified.

The above analysis tells us where to look for the new contributions. Wherever $\partial f_{0} / \partial \epsilon$ is involved, we should examine the corresponding terms where $\partial f_{0} / \partial \epsilon$ is replaced by $\partial(\delta f) / \partial \epsilon$, or $\left(\partial f_{1} / \partial \epsilon\right) \hat{\tau}_{z}$, to see whether they may not really be negligible. Furthermore, if $(\partial A / \partial \epsilon)(\partial B / \partial t)$ is not negligible, then probably $(\partial A / \partial t)(\partial B / \partial \epsilon)$ is also not negligible. So there are many possibilities to be considered. There are three places where such new terms may be found:

(i) In the last two terms of Eq. (6.1), which we have so far neglected (unduly). The new terms arise by taking the leading nonvanishing contribution to $\hat{Y}$, namely, $\left[-i \hat{\Delta}, f_{1} \hat{\tau}_{z}\right]$, which gives the following contributions to Eqs. (6.6) and (6.7): To the left-hand side of Eq. (6.6), one must add

$$
-\left\{\tilde{N}_{3 \cdot}(\operatorname{Re} \Delta) f_{1}\right\}_{\epsilon, t}+\left\{\tilde{N}_{2},(\operatorname{Im} \Delta) f_{1}\right\}_{\epsilon, t} .
$$

and to the left-hand side of Eq. (6.7), one must add

$$
-\left\{\tilde{R}_{3},(\operatorname{Im} \Delta) f_{1}\right\}_{\epsilon, t}-\left\{\tilde{R}_{2},(\operatorname{Re} \Delta) f_{1}\right\}_{\epsilon, t} .
$$

(ii) In evaluating Eq. (6.5), we have so far unduly neglected

$$
\begin{aligned}
& \frac{1}{2} i\left\{-i \hat{\Delta}, \delta f+f_{1} \hat{\tau}_{z}\right\}_{\epsilon, t} \\
& -\frac{1}{2} i\left\{\delta f+f_{1} \hat{\tau}_{z},-i \hat{\Delta}\right\}_{\epsilon, t}=-\left(\partial_{\epsilon} \delta f\right) \dot{\hat{\Delta}}
\end{aligned}
$$

Thus to the left-hand side of Eq. (6.6) one must add

$$
\left(\tilde{R}_{2} \operatorname{Re} \dot{\Delta}+\tilde{R}_{3} \operatorname{Im} \dot{\Delta}\right) \partial_{\epsilon}(\delta f) \text {. }
$$

and to the left-hand side of Eq. (6.7) one must add

$$
\left(\tilde{N}_{3} \operatorname{Re} \dot{\Delta}-\tilde{N}_{2} \operatorname{Im} \dot{\Delta}\right) \partial_{\epsilon}(\delta f) .
$$

(iii) Toward the end of Eq. (7.2), we have neglected a $(\delta f)$ and a $f_{1}$ contribution. They give the following additions to Eq. (7.4):

$$
\frac{1}{2} \int_{0}^{\infty} d \epsilon\left(\left\{\tilde{N}_{2}+i \tilde{N}_{3}, \delta f\right\}_{\epsilon, t}-i\left\{\tilde{R}_{2}+i \tilde{R}_{3}, f_{1}\right\}_{\epsilon, t}\right)
$$

These terms are actually smaller by a factor $\eta$ than the terms originally kept in Eq. (7.4), because of the "change-of-order-by-integration" concept explained earlier. However, we will keep these terms in the equation for $\Delta$, in order to obtain a deliberately "under simplified" set of equations for a purpose already explained before.

So far the new contributions are not gauge invariant. This deficiency may be partially removed by replacing $f_{1}$ everywhere in Eqs. (9.2)-(9.6) by $\left(f_{1}+e \phi f_{0, \epsilon}\right)$. [The $e \phi$ terms are of order $\omega^{2}$, and can be rigorously obtained only by extending Eq. (4.2) to the next order. This will be a very tedious task, so we will follow the present heuristic approach instead.] One does not need to worry about a possible nonuniqueness of this approach, because the difference between $f_{1}+e \phi f_{0, \epsilon}$ and any other gauge invariant combination involving $f_{1}$, say $f_{1}-\frac{1}{2} \dot{\chi} f_{0, \epsilon}$, would be negligibly small when inserted into Eqs. (9.2)-(9.6). The replacement prescription introduced here does not yet make the new contributions completely invariant with respect to the gauge transformation introduced so far. It turns out that the gauge property of $f_{1}$ must still be modified - a point which will soon be made more explicit. In the above evaluation of the new contributions, I have ignored several higher-order contributions, such as

$$
\frac{1}{2} i\left\{i e \phi, \delta f+f_{1} \hat{\tau}_{z}\right\}_{\epsilon, t}-\frac{1}{2} i\left\{\delta f+f_{1} \hat{\tau}_{z}, i e \phi\right\}_{\epsilon, t}
$$

[which belongs originally to the expression just above Eq. (9.4)], because these terms are of order $\eta^{3}|\Delta|$ for 
$\epsilon \leq|\Delta|$, while the terms kept so far are all of order $\geq \eta^{2}|\Delta|$, and also because these terms are not needed to make a lower-order contribution gauge invariant.

Equations (9.2)-(9.6) may be transformed into the real- $\Delta$ gauge by using Eq. (4.8). The results are

(i) To the left-hand side of Eq. (6.16), one must add

$$
\begin{aligned}
& -\left\{N_{3},|\Delta|\left(f_{1}+e \phi f_{0, \epsilon}\right)\right\}_{\epsilon, t}+R_{2}|\dot{\Delta}| \partial_{\boldsymbol{\epsilon}}(\delta f) \\
& \quad+|\Delta| \dot{\chi} \partial_{\boldsymbol{\epsilon}}\left[N_{2}\left(f_{1}+e \phi f_{0, \epsilon}\right)\right]+|\Delta| \dot{\chi} R_{3} \partial_{\boldsymbol{\epsilon}}(\delta f)
\end{aligned}
$$

(ii) To the left-hand side of Eq. (6.17), one must add

$$
\begin{aligned}
-\left\{R_{2},|\Delta|\left(f_{1}+e \phi f_{0, \epsilon}\right)\right\}_{\epsilon, t}+N_{3}|\dot{\Delta}| \partial_{\epsilon}(\delta f) \\
-|\Delta| \dot{\chi} \partial_{\epsilon}\left[R_{3}\left(f_{1}+e \phi f_{0, \epsilon}\right)\right]-|\Delta| \dot{\chi} N_{2} \partial_{\epsilon}(\delta f) .
\end{aligned}
$$

(iii) To the left-hand side of Eq. (7.8), one must add

$\frac{1}{2} \int_{0}^{\infty} d \epsilon\left[\left\{N_{2}+i N_{3}, \delta f\right\}_{\epsilon, t}-i\left\{R_{2}+i R_{3}, f_{1}+e \phi f_{0, \epsilon}\right\}_{\epsilon, t}-i \dot{\chi}\left(N_{2}+i N_{3}\right) \partial_{\epsilon} \delta f-\dot{\chi}\left(R_{2}+i R_{3}\right) \partial_{\epsilon}\left(f_{1}+e \phi f_{0, \epsilon}\right)\right]$

Let us now examine the effect of these new contributions to the local charge conservation law. Instead of Eq. (9.1), we now have [with $\bar{f}_{1}$ denoting $\left.\left(f_{1}+e \phi f_{0, \epsilon}\right)\right]$ :

$$
\begin{aligned}
v \equiv & (\dot{\rho}+\vec{\nabla} \cdot \vec{j}) / 2 e N(0) \\
=- & \int_{0}^{\infty} d \epsilon\left(\dot{N}_{1} \bar{f}_{1}+|\Delta|\left[\left\{N_{3}, \delta f\right\}_{\epsilon, t}-\left\{R_{2} \bar{f}_{1}\right\}_{\epsilon, t}-N_{2} \dot{\chi} \partial_{\boldsymbol{\epsilon}}(\delta f)-R_{3} \dot{\chi} \partial_{\boldsymbol{\epsilon}} \bar{f}_{1}\right]+\left\{R_{2},|\Delta| \bar{f}_{1}\right\}_{\epsilon, t}\right. \\
& \left.\quad-N_{3}|\dot{\Delta}| \partial_{\boldsymbol{\epsilon}}(\delta f)+|\Delta| \dot{\chi} \partial_{\boldsymbol{\epsilon}}\left(R_{3} \bar{f}_{1}\right)+N_{2}|\Delta| \dot{\chi} \partial_{\boldsymbol{\epsilon}}(\delta f)\right) \\
= & -\int_{0}^{\infty} d \epsilon\left\{\left[\dot{N}_{1}+\left(\partial_{\boldsymbol{\epsilon}} R_{2}\right)|\dot{\Delta}|+\left(\partial_{\boldsymbol{\epsilon}} R_{3}\right)|\Delta| \dot{\chi}\right] \bar{f}_{1}+\partial_{t}\left[N_{3}|\Delta| \partial_{\boldsymbol{\epsilon}}(\delta f)\right]\right\} .
\end{aligned}
$$

We now show that these terms are indeed negligibly small. Recalling that we have so far systematically neglected terms of order $D k^{2} \omega$, and since all terms in Eq. (9.10) are already proportional to $\omega$, the spectral quantities in this equation must be regarded as satisfying Eq. (5.13) with its left-hand-side $D k^{2}$ terms neglected. ${ }^{27}$ Within this approximation, one can easily show that $\gamma \simeq 0$. Also, in the same approximation, I have previously established Eq. (5.10) (cf. Appendix A), which reduces, in the real- $\Delta$ gauge, simply to $\dot{\alpha}=i\left(\partial_{\epsilon} \beta\right)|\dot{\Delta}|$. In terms of the notations $N_{i}$ and $R_{i}$ [cf. Eq. (5.14)], we thus have established that for Eq. (9.10), we may put

$N_{3} \simeq R_{3} \simeq 0, \quad \dot{N}_{1} \simeq-\left(\partial_{\epsilon} R_{2}\right)|\dot{\Delta}|, \dot{R}_{1} \simeq\left(\partial_{\epsilon} N_{2}\right)|\dot{\Delta}|$,

which changes the right-hand side of Eq. (9.10) to zero. It is therefore consistent with our approximation of neglecting all terms quadratic in $\omega$ and/or $D k^{2}$, to use Eq. (9.11) to simplify Eqs. (9.7)-(9.9). We thus conclude that

(i)' To the left-hand side of Eq. (6.16), one must now add

$$
R_{2}|\dot{\Delta}| \partial_{\epsilon}(\delta f)+|\Delta| \dot{\chi} \partial_{\epsilon}\left[N_{2}\left(f_{1}+e \phi f_{0, \epsilon}\right)\right] .
$$

(ii)' To the left-hand side of Eq. (6.17), one must now add

$$
\begin{aligned}
\dot{N}_{1}\left(f_{1}+e \phi f_{0, \epsilon}\right)-|\Delta|\left\{R_{2}, f_{1}+e \phi f_{0, \epsilon}\right\}_{\epsilon, t} \\
-|\Delta| \dot{x} N_{2} \partial_{\epsilon}(\delta f) .
\end{aligned}
$$

(iii)' To the left-hand side of Eq. (7.8), one must now add

$$
\begin{aligned}
\frac{1}{2} \int_{0}^{\infty} d \epsilon[ & \left\{N_{2}, \delta f\right\}_{\epsilon, t}-i\left\{R_{2}, f_{1}+e \phi f_{0, \epsilon}\right\}_{\epsilon, t} \\
& \left.-i \dot{\chi} N_{2} \partial_{\epsilon} \delta f-\dot{\chi} R_{2} \partial_{\epsilon}\left(f_{1}+e \phi f_{0, \epsilon}\right)\right] .
\end{aligned}
$$

The local charge conservation law is now satisfied essentially exactly [except for the approximations made in Eq. (8.5)]. Unfortunately, these expressions are still not invariant under the gauge transformation defined so far, and the solution cannot be found by adding to them negligible terms of higher orders. For example, to make the term $|\Delta| \dot{\chi} N_{2} \partial_{\mathbf{\epsilon}}(\delta f)$ of Eq. (9.13) "gauge invariant" (in the sense used so far), one must add to it either $|\Delta| 2 e \phi N_{2} \partial_{\epsilon}(\delta f)$, or $|\Delta|\left(-2 f_{1} / f_{0, \epsilon}\right) N_{2} \partial_{\epsilon}(\delta f)$. Neither of these are of higher order than the terms kept. Furthermore, such terms cannot possibly arise from the present theory, simply because in the method of LO, $f_{0, \epsilon}$ never appears in the denominator, and $\partial_{\epsilon}$ always appears together with $\partial_{t}$. The solution, therefore, lies in a modification of the gauge transformation used so far, particularly the transformation property assigned for $f_{1}$. To understand this, let us go back to the unabridged Eqs. (9.7)-(9.9). It is easy to understand the last terms in Eqs. (9.7)-(9.8). Had they been the only $\dot{\chi}$-dependent terms in these equations, we would combine them with the last terms in the lefthand side of Eqs. (6.16) and (6.17), and conclude that $2 f_{1}-\dot{\chi} \partial_{\epsilon}\left(f_{0}+\delta f\right)$ or

$$
2\left[f_{1}+e \phi \partial_{\epsilon}\left(f_{0}+\delta f\right)\right]-(2 e \phi+\dot{\chi}) \partial_{\epsilon}\left(f_{0}+\delta f\right)
$$


must be gauge invariant. I try to understand this as follows: If a "true distribution" of quasiparticles ${ }^{28}$

$$
f_{\text {true }}(\epsilon)=\frac{1}{2}\left[1-f_{0}(\epsilon)-\delta f(\epsilon)-f_{1}(\epsilon)\right]
$$

is subjected to a small uniform scalar potential $e \phi$, it seems that it should simply change to ${ }^{29}$ :

$$
\begin{aligned}
f_{\text {true }}(\epsilon+e \phi)= & \frac{1}{2}\left[1-f_{0}(\epsilon)-\delta f(\epsilon)-f_{1}(\epsilon)\right] \\
& -\frac{1}{2} e \phi \partial_{\epsilon}\left(f_{0}+\delta f+f_{1}\right),
\end{aligned}
$$

so that the even and odd parts of the gauge-invariant combinations should simply be, respectively,

$$
f_{1}+e \phi \partial_{\mathbf{\epsilon}}\left(f_{0}+\delta f\right)
$$

and

$$
\delta f+e \phi \partial_{\epsilon}\left(f_{1}\right)
$$

Unfortunately, the second to the last terms in Eqs. (9.7) and (9.8) cannot be understood this way, so the exact gauge properties of $f_{1}$ (and perhaps $\delta f$ ) for $\epsilon \leq|\Delta|$ remain a puzzle to me. [Only for $\epsilon \leq|\Delta|$ are $\partial_{\epsilon}(\delta f)$ and $\partial_{\epsilon} f_{1}$ of the same order as $\partial_{\epsilon} f_{0}$. For $\epsilon>|\Delta|$, there is still no problem.] In the following, I propose an approximate treatment of Eqs. (9.12)-(9.14), which will circumvent this difficulty.

Recalling the remark made earlier that $f_{1}+e \phi f_{0,}$. is actually of order $\eta^{2}$ for $\epsilon \leq|\Delta|,{ }^{26}$ we propose to neglect the $\dot{x}$ term in Eq. (9.12). The corresponding $\dot{\chi}$ term in Eq. (9.13) cannot be so neglected. So we propose to replace this $\dot{x}$ by $\dot{x}+2 e \phi$. This is equivalent to replacing the first term in Eq. (9.15) by $2\left(f_{1}+e \phi f_{0, \epsilon}\right)$, which is also equivalent to replacing $\left(f_{1}+e \phi f_{0, \epsilon}\right)$ everywhere else by $\left[f_{1}+e \phi \partial_{\epsilon}\right.$ $\left.\times\left(f_{0}+\delta f\right)\right]$, and $e \dot{\overrightarrow{\mathrm{A}}} f_{0, \mathrm{e}}$ everywhere by

$e \dot{\overrightarrow{\mathrm{A}}} \partial_{\epsilon}\left(f_{0}+\delta f\right)$. But note that the former is an unknown function of $\epsilon$ yet to be solved from Eq. (6.17), and therefore it may be identified as to have any relation with another unknown function $f_{1}$, while the latter replacement involves changes only in the negligible order. The corresponding change to Eq. (9.14) is to replace its last two terms by $-i(\dot{x}+2 e \phi) N_{2} \partial_{\epsilon}(\delta f)$. Such changes obviously do not affect our proof that $\dot{\rho}+\vec{\nabla} \cdot \vec{j}=0$, but they have reestablished an invariance for the set of TDGL equations with respect to the simple gauge transformation:

$$
\begin{aligned}
& \phi \rightarrow \phi-\dot{\Lambda}, \quad \overrightarrow{\mathrm{A}} \rightarrow \overrightarrow{\mathrm{A}}+\vec{\nabla} \Lambda \\
& x \rightarrow x+2 e \Lambda, \quad f_{1} \rightarrow f_{1}+e \dot{\Lambda} f_{0, \epsilon}
\end{aligned}
$$

The final set of equations so derived is summarized in the next section, together with a simplified version which assumes (i) $\gamma=0$, and (ii) an approximate charge neutrality condition. In that section we shall also show that the remaining new terms arising from the discovery made in this section do make important contributions.

\section{SUMMARY AND DISCUSSION}

The new set of TDGL equations derived in this work, which includes the more important new contributions found in Sec. IX, may be summarized as follows (in the real- $\Delta$ gauge):

$$
\begin{aligned}
& \bar{A} \Delta-\bar{B}|\Delta|^{2} \Delta-C\left(\dot{\Delta}+2 i e \phi \Delta-D \vec{D}^{2} \Delta\right)+(\Delta /|\Delta|) \int_{0}^{\infty} d \epsilon\left[\left(R_{2}+i R_{3}\right) \delta f+i\left(N_{2}+i N_{3}\right)\left(f_{1}+e \phi f_{0, \epsilon}\right)\right. \\
& +\frac{1}{2}\left\{N_{2}, \delta f\right\}_{\epsilon, t}-\left(\frac{1}{2} i\right)\left\{R_{2}, f_{1}+e \phi . f_{0, \epsilon}\right\}_{\epsilon, t} \\
& \left.-i(\dot{\chi}+2 e \phi) N_{2} \partial_{\epsilon}(\delta f)\right]=0 \\
& \overrightarrow{\mathrm{j}}=2\left(\sigma_{0} / e\right) C|\Delta|^{2} \overrightarrow{\mathrm{Q}}+\left(\sigma_{0} / e\right) \int_{0}^{\infty} d \epsilon\left\{\left[\left(N_{2} \vec{\nabla} R_{3}+R_{2} \vec{\nabla} N_{3}-N_{3} \vec{\nabla} R_{2}-R_{3} \vec{\nabla} N_{2}\right)\right.\right. \\
& \left.+2 \vec{Q}\left(N_{2} R_{2}+N_{3} R_{3}\right)\right] \delta f+\left(N_{2} R_{3}-R_{2} N_{3}\right) \vec{\nabla} \delta f \\
& \left.+\left(N_{1}^{2}+N_{2}^{2}+N_{3}^{2}\right)\left(\vec{\nabla} f_{1}-e \dot{\overrightarrow{\mathrm{A}}} f_{0, \mathrm{e}}\right)\right\} \\
& \rho=-2 e N(0) \int_{0}^{\infty} d \epsilon N_{1}\left(f_{1}+e \phi f_{0, \epsilon}\right) \\
& \frac{1}{2} D\left[\alpha(\vec{\nabla} \pm i \vec{Q})^{2}(\beta \pm i \gamma)-(\beta \pm i \gamma) \vec{\nabla}^{2} \alpha\right]=\left(-i \epsilon+\frac{1}{2} \tau_{E}^{-1}\right)(\beta \pm i \gamma)-|\Delta| \alpha+\tau_{s}^{-1} \alpha(\beta \pm i \gamma)
\end{aligned}
$$


with

$$
\begin{aligned}
& \begin{array}{l}
\alpha \equiv N_{1}+i R_{1}, \quad \beta=N_{2}+i R_{2}, \quad \gamma=N_{3}+i R_{3}, \quad \alpha^{2}+\beta^{2}+\gamma^{2}=1 \\
-D \vec{\nabla} \cdot\left[\left(N_{1}^{2}-R_{2}^{2}-R_{3}^{2}\right) \vec{\nabla} \delta f\right]+D\left(N_{2} R_{3}-N_{3} R_{2}\right) \vec{\nabla} \cdot\left(\vec{\nabla} f_{1}-e \dot{\overrightarrow{\mathrm{A}}} f_{0, \epsilon}\right) \\
-2 D\left[\left(R_{2} \vec{\nabla} N_{3}-R_{3} \vec{\nabla} N_{2}\right)+\overrightarrow{\mathrm{Q}}\left(N_{2} R_{2}+N_{3} R_{3}\right)\right] \cdot\left(\vec{\nabla} f_{1}-e \dot{\overrightarrow{\mathrm{A}}} f_{0, \epsilon}\right) \\
\quad+R_{2}|\dot{\Delta}| \partial_{\epsilon}\left(f_{0}+\delta f\right)+N_{1}\left(\partial_{,}+\tau_{\bar{E}}^{-1}\right) \delta f-R_{3}|\Delta|\left(2 f_{1}-\dot{\chi} f_{0, \epsilon}\right)=0 ;
\end{array}
\end{aligned}
$$

and

$$
\begin{aligned}
-D \vec{\nabla} \cdot[ & \left.\left(N_{1}^{2}+N_{2}^{2}+N_{3}^{2}\right)\left(\vec{\nabla} f_{1}-e \dot{\overrightarrow{\mathrm{A}}} f_{0, \epsilon}\right)\right]-D\left(N_{2} R_{3}-N_{3} R_{2}\right) \vec{\nabla} \delta f \\
& -2 D\left[\left(N_{2} \vec{\nabla} R_{3}-N_{3} \vec{\nabla} R_{2}\right)+\overrightarrow{\mathrm{Q}}\left(N_{2} R_{2}+N_{3} R_{3}\right)\right] \cdot \vec{\nabla} \delta f+N_{3}|\dot{\Delta}| f_{0, \epsilon}+\left(\partial_{\ell}+\tau_{E}^{-1}\right)\left[N_{1}\left(f_{1}+e \phi f_{0, \epsilon}\right)\right] \\
& +N_{2}|\Delta|\left(2 f_{1}-\dot{\chi} f_{0, \epsilon}\right)-|\Delta|\left\{R_{2}, f_{1}+e \phi f_{0, \epsilon}\right\}_{\epsilon, t}-|\Delta|(\dot{\chi}+2 e \phi) N_{2} \partial_{\epsilon}(\delta f) \\
& =\tau_{E}^{-1} f_{0, \epsilon} \int_{0}^{\infty} N_{1}\left(\epsilon^{\prime}\right)\left[f_{1}\left(\epsilon^{\prime}\right)+e \phi f_{0, \epsilon^{\prime}}\left(\epsilon^{\prime}\right)\right] d \epsilon^{\prime} .
\end{aligned}
$$

The above set of TDGL equations satisfies an exact gauge invariance [with respect to the gauge transformation defined in Eq. (9.16)], and an almost exact local charge conservation law $\dot{\rho}+\vec{\nabla} \cdot \vec{j} \simeq 0$ (with a percentage error in the order $|\Delta|^{2} / T^{2}$ ). Its derivation is based on a single condition $|\Delta|<<T$, which is equivalent to $1-T / T_{c}<<1$. The set of equations is therefore valid for all intrinsic dynamic processes and instabilities, which may involve a finite deviation from thermal equilibrium, and a nontrivial spatial dependence. Imposed spatial and/or temporal variations are also allowed, if only they involve time scales not shorter than $\sim(T /|\Delta|)(\hbar /|\Delta|)$, and length scales not shorter than $\sim \hbar v_{F} /|\Delta|$. The system is assumed to have a large amount of ordinary impurities to put the system in the "dirty limit" ( $\tau T<<1)$. Magnetic impurities are also allowed, at any amount which may or may not drive the system gapless. Only electromagnetic fields have been included as external driving forces, so the system of equations must still be extended, if it is to include such additional driving forces as phonon injection, or quasiparticle injection via tunneling processes. ${ }^{30}$

The above set of TDGL equations may be further simplified under the following approximations:

(a) Equation (5.17) may be solved approximately by neglecting the spatial gradients of $\gamma$, giving

$$
\gamma=\frac{\frac{1}{2} D(\alpha / \beta) \vec{\nabla} \cdot\left(Q \beta^{2}\right)}{-i \epsilon+\left(2 \tau_{E}\right)^{-1}+\tau_{s}^{-1} \alpha+\frac{1}{2} D Q^{2} \alpha},
$$

which shows that $\gamma \equiv N_{3}+i R_{3}$ is smaller than $\beta \equiv N_{2}+i R_{2}$ by roughly a factor $|\Delta| / T$. Thus unless one is interested in studying physical phenomena which are due solely to a nonvanishing $\gamma$, otherwise one may put $N_{3} \simeq R_{3} \simeq 0$.

(b) In terms of $\bar{f} \equiv f_{1}+e \phi f_{0, \epsilon}$, we may rewrite the first term of Eq. (10.7) as

$$
-D \vec{\nabla} \cdot\left(M^{(T)} \vec{\nabla} \bar{f}_{1}\right)-e D \vec{\nabla} \cdot\left(M^{(T)} \overrightarrow{\mathrm{E}}\right) f_{0, \epsilon}
$$

where $M^{(T)} \equiv N_{1}^{2}+N_{2}^{2}+N_{3}^{2}$. Approximating $M^{(T)}$ by 1 , and using $\vec{\nabla} \cdot \vec{E}=4 \pi \rho$, we may estimate the second term of Eq. (10.8) as

$$
-\left(D \lambda_{\mathrm{TF}}^{-2}\right) f_{0, \epsilon} \int_{0}^{\infty} N_{1}\left(\epsilon^{\prime}\right) \bar{f}_{1}\left(\epsilon^{\prime}\right) d \epsilon^{\prime}
$$

where $\lambda_{\mathrm{TF}} \equiv\left[8 \pi N(0) e^{2}\right]^{-1 / 2}$ is the Thomas-Fermi (TF) wavelength. Since $D \lambda_{\mathrm{TF}}^{-2} \sim \tau \epsilon_{F}^{2}$ is always much bigger than $\tau_{E}^{-1}$, it follows that the right-hand-side term of Eq. (10.7) may be always neglected. Since this analysis also establishes that $\rho \sim e N(0)\left(\lambda_{\mathrm{TF}} / \xi_{\mathrm{BCS}}\right)^{2} \eta^{3} \Delta$ is much smaller than its expected order $e N(0) \eta \Delta$, we may call this approximation "the approximate charge neutrality condition." 31

Applying both approximation (a) and (b) to Eqs. $(10.1)-(10.7)$, we obtain the following simplified set of equations:

$$
\begin{aligned}
\bar{A} \Delta- & \bar{B}|\Delta|^{2} \Delta-C\left(\dot{\Delta}+2 i e \phi \Delta-D D^{2} \Delta\right) \\
& +(\Delta /|\Delta|) \int_{0}^{\infty} d \epsilon\left[R_{2} \delta f+i N_{2}\left(f_{1}+e \phi f_{0, \epsilon}\right)+\frac{1}{2}\left\{N_{2}, \delta f\right\}_{\epsilon, t}-\frac{1}{2} i\left\{R_{2}, f_{1}+e \phi f_{0, \epsilon}\right\}_{\epsilon, t}-i(\dot{\chi}+2 e \phi) N_{2} \partial_{\epsilon}(\delta f)\right]=0 ;
\end{aligned}
$$

$j=\left(\sigma_{0} / e\right)\left(2 C|\Delta|^{2} \overrightarrow{\mathrm{Q}}+\int_{0}^{\infty} d \epsilon\left[2 N_{2} R_{2} \overrightarrow{\mathrm{Q}} \delta f+\left(N_{1}^{2}+N_{2}^{2}\right)\left(\vec{\nabla} f_{1}-e \dot{\overrightarrow{\mathrm{A}}} f_{0, \epsilon}\right)\right]\right) ;$ 


$$
\begin{aligned}
& \rho=-2 e N(0) \int_{0}^{\infty} d \epsilon N_{1}\left(f_{1}+e \phi f_{0, \epsilon}\right) ; \\
& \frac{1}{2} D\left[\alpha\left(\nabla^{2}-Q^{2}\right) \beta-\beta \nabla^{2} \alpha\right]=\left(-i \epsilon+\frac{1}{2} \tau_{E}^{-1}\right) \beta-|\Delta| \alpha+\tau_{s}^{-1} \alpha \beta ; \\
& \alpha=N_{1}+i R_{1}, \quad \beta=N_{2}+i R_{2}, \quad \alpha^{2}+\beta^{2}=1 ; \\
& -D \vec{\nabla} \cdot\left[\left(N_{1}^{2}-R_{2}^{2}\right) \vec{\nabla} \delta f\right]-2 D N_{2} R_{2} \vec{Q} \cdot\left(\vec{\nabla} f_{1}-e \dot{\vec{A}} f_{0, \epsilon}\right)+R_{2}|\dot{\Delta}| \partial_{\epsilon}\left(f_{0}+\delta f\right)+N_{1}\left(\partial_{t}+\tau_{E}^{-1}\right) \delta f=0 ;
\end{aligned}
$$

and

$$
\begin{aligned}
-D \vec{\nabla} \cdot\left[\left(N_{1}^{2}+N_{2}^{2}\right)\left(\vec{\nabla} f_{1}-e \dot{\overrightarrow{\mathrm{A}}} f_{0, \epsilon}\right)\right]-2 D N_{2} R_{2} \overrightarrow{\mathrm{Q}} \cdot \vec{\nabla} \delta f+\left(\partial_{t}+\tau_{\bar{E}}^{-1}\right)\left[N_{1}\left(f_{1}+e \phi f_{0, \epsilon}\right)\right] \\
+N_{2}|\Delta|\left(2 f_{1}-\dot{\chi} f_{0, \epsilon}\right)-|\Delta|\left\{R_{2}, f_{1}+e \phi f_{0, \epsilon}\right\}_{\epsilon, t}-|\Delta|(\dot{\chi}+2 e \phi) N_{2} \partial_{\epsilon}(\delta f)=0 .
\end{aligned}
$$

This set of TDGL equations appears now very similar to the set presented previously, ${ }^{14,17}$ but there are still some essential differences due to the new contributions found in Sec. IX of this work. To convince the readers that the new contributions are indeed important in the energy range $\epsilon \leq|\Delta|$, let us look at the following simplified version of Eq. (10.15), which is sufficient for illustrating the essential point that I wish to make:

$$
N_{1} \partial_{t}(\delta f)=-R_{2}|\dot{\Delta}| \partial_{\epsilon}\left(f_{0}+\delta f\right) .
$$

In the BCS limit, when $R_{2} / N_{1}=|\Delta| / \epsilon$, this equation may be solved exactly, giving

$$
\begin{aligned}
\left(f_{0}+\delta f\right)(\epsilon, t)=\left(f_{0}+\delta f\right)(\operatorname{sgn}(\epsilon) & {\left[\epsilon^{2}-|\Delta(t)|^{2}\right.} \\
& \left.\left.+|\Delta(0)|^{2}\right]^{1 / 2}, 0\right) .
\end{aligned}
$$

On the other hand, if one omits from Eq. (10.17) the term $R_{2}|\dot{\Delta}| \partial_{e}(\delta f)$, which is one of the new contributions found in Sec. IX, then the solution in the same BCS limit becomes

$$
\delta f(\epsilon, t)=f_{0, \epsilon}\left[|\Delta(0)|^{2}-|\Delta(t)|^{2}\right] / 2 \epsilon+\delta f(\epsilon, 0) .
$$

It is clear that the difference between Eq. (10.18) and Eq. (10.19) is finite for $\epsilon \leq|\Delta|$, and that Eq. (10.18) is a more physical solution than Eq. (10.19) in this energy range.

The inclusion of inelastic collision effects [i.e., the term $N_{1} \tau_{E}^{-1} \delta f$ of Eq. (10.15)] in Eq. (10.17) already makes this equation difficult to solve exactly, but it should be clear from order-of-magnitude analysis that the new contributions become relatively unimportant only if $\omega \tau_{E}<<1$ is assumed. [If the condition is made even stronger to $\tau_{E}|\Delta|<<1$, then the system becomes gapless, and the only important energy scale becomes $\epsilon \sim\left(T\right.$ and $\left.\tau_{E}^{-1}\right) \gg|\Delta|$. The new contributions are then obviously unimportant, along with many "old contributions," so that the TDGL equations can be greatly simplifed in this case. This temperature range, however, is usually too narrow to have much physical interest.]

Finally, it should be noted that the new terms discovered in Sec. 1X are important only for nonlinear dynamic processes [e.g., phase slip processes outside the "local-equilibrium regime" as defined by Ref. 14, Eq. (8); or nonlinear flux flow, which can lead to a dissipative phase transition ${ }^{32}$ ]. For linear transport or instability analysis, and when a nonvanishing $\gamma$ is not crucial for correctly describing the physics involved, it is still adequate to use the set of TDGL equations derived previously, ${ }^{14,17}$ to which the present set reduces in this limit.

\section{ACKNOWLEDGMENTS}

This work began when the author visited Institut für Theorie der Kondensierten Materie of the University of Karlsruhe. The author is very grateful to Professor A. Schmid for his invitation, hospitality, financial support during that period, and for explaining the essentials of the Larkin-Ovchinnikov method. Many very stimulating and informative conversations also took place there with R. J. Watts-Tobin, and to a lesser extent, with L. Kramer and U. Eckern, to all of whom I wish to express appreciation. This work is supported by the NSF under Grants No. DMR 7681328 and No. DMR 78-21968.

\section{APPENDIX A}

In this Appendix we show that Eq. (5.10) follows from Eqs. (5.7) and (5.8), if the left-hand sides of the latter equations are neglected. For $\tau_{s}^{-1}=0$, this proof may be simply achieved by employing the explicit solutions of Eqs. (5.7) and (5.8) under the stated approximation. For $\tau_{s}^{-1} \neq 0$, the proof is less trivial, which I give below (for the retarded case only).

Equations (5.7) and (5.8) without their left-hand sides read

$$
\begin{aligned}
{\left[\tau_{s}^{-1}(\tilde{\beta} \pm i \tilde{\gamma})-\Delta\right.} & \left.\left(\text { or } \Delta^{*}\right)\right] \alpha \\
& =-\left(-i \epsilon+\frac{1}{2} \tau_{E}^{-1}\right)(\tilde{\beta} \pm i \tilde{\gamma}) .
\end{aligned}
$$


Differentiating this equation with respect to $t$ gives

$$
\begin{aligned}
& \left(-i \epsilon+\frac{1}{2} \tau_{E}^{-1}+\tau_{s}^{-1} \alpha\right)(\dot{\tilde{\beta}} \pm i \dot{\tilde{\gamma}}) \\
& \quad+\left[\tau_{s}^{-1}(\tilde{\beta} \pm i \tilde{\gamma})-\Delta\right] \dot{\alpha}=\alpha \dot{\Delta}\left(\text { or } \dot{\Delta}^{*}\right)
\end{aligned}
$$

Multiplying Eq. (A2) by $(\tilde{\beta} \mp i \tilde{\gamma}) \alpha$, substituting Eq. (A1) in it, adding up the two cases (i.e., the choices of signs in \pm or $\mp$ ), and using $\alpha^{2}+(\beta+i \gamma)(\beta-i \gamma)=1$, gives

$$
\dot{\alpha}=-\frac{\alpha^{2}\left[(\tilde{\beta}-i \tilde{\gamma}) \dot{\Delta}+(\tilde{\beta}+i \tilde{\gamma}) \dot{\Delta}^{*}\right]}{2\left(-i \epsilon+\frac{1}{2} \tau_{E}^{-1}+\tau_{s}^{-1} \alpha^{3}\right)} .
$$

Now differentiating Eq. (A1) with respect to $\epsilon$ gives

$$
\begin{aligned}
& \left(-i \epsilon+\frac{1}{2} \tau_{E}^{-1}+\tau_{s}^{-1} \alpha\right) \partial_{\epsilon}(\tilde{\beta} \pm i \tilde{\gamma}) \\
& \quad-\alpha^{-1}\left(-i \epsilon+\frac{1}{2} \tau_{E}^{-1}\right)(\tilde{\beta} \pm i \tilde{\gamma}) \partial_{\epsilon} \alpha=i(\tilde{\beta} \pm i \tilde{\gamma}),
\end{aligned}
$$

which implies

$$
(\tilde{\beta}-i \tilde{\gamma}) \partial_{\mathbf{\epsilon}}(\tilde{\beta}+i \tilde{\gamma})=(\tilde{\beta}+i \tilde{\gamma}) \partial_{\mathbf{\epsilon}}(\tilde{\beta}-i \tilde{\gamma}) .
$$

Then using

$$
\begin{aligned}
\partial_{\epsilon} \alpha & =-\frac{1}{2} \alpha^{-1} \partial_{\epsilon}[(\tilde{\beta}+i \tilde{\gamma})(\tilde{\beta}-i \tilde{\gamma})] \\
& =-\alpha^{-1}(\tilde{\beta} \mp i \tilde{\gamma}) \partial_{\epsilon}(\tilde{\beta} \pm i \tilde{\gamma})
\end{aligned}
$$

in Eq. (A4), I obtain

$$
\partial_{\epsilon}(\tilde{\beta} \pm i \tilde{\gamma})=\frac{i \alpha^{2}(\tilde{\beta} \pm i \tilde{\gamma})}{-i \epsilon+\frac{1}{2} \tau_{E}^{-1}+\tau_{s}^{-1} \alpha^{3}}
$$

Combining Eqs. (A3) and (A7) gives finally Eq. (5.10) in the main text.

Changing the signs of all the $\tau_{E}^{-1}$ terms converts the retarded case to the advanced case, which also follows from the symmetry relations in Eq. (5.11).

\section{APPENDIX B}

In this Appendix I show that the collision integrals given in Eqs. (6.8) - (6.11) may be reduced to Eqs. (6.12) and (6.15), simply by invoking the small parameter $|\Delta| / T$ in the Ginzburg-Landau regime, without the need of employing such an idea as "multilated collision operator."15 (At lower temperatures, this idea would be needed to reduce the collision integrals, but it would be less accurate.)

The proof is very similar to the reasoning which $\mathrm{I}$ used to simplify Eq. (5.2) to Eqs. (5.5) and (5.6). First I note that the dominant contributions to the $\epsilon_{1}$ integrals in Eqs. (6.8)-(6.11) are from the region where $\omega(\overrightarrow{\mathrm{k}}) \simeq C_{0} P_{F} \theta \sim \epsilon_{1} \sim T$. In this energy range $\alpha \sim 1, \tilde{\beta}, \tilde{\gamma} \sim \eta$, so the collision integrals may be reduced to

$$
\begin{aligned}
& K_{12} \simeq K_{21} \simeq 0, \\
& K_{11}[\delta f]=-\frac{1}{4} i N(0) g^{2} N_{1}(\epsilon) \int d \Omega_{\hat{p}_{1}} \int \frac{d \epsilon_{1}}{2 \pi}\left[D_{\hat{P} \cdot \hat{p}_{1}}^{R}\left(\epsilon_{1}\right)-D_{\hat{P} \cdot \hat{p}_{1}}^{A}\left(\epsilon_{1}\right)\right]\left[\sinh \left(\frac{\epsilon_{1}}{2 T}\right) \cosh \left(\frac{\epsilon_{1}}{2 T}\right)\right]^{-1} \\
& \times\left[\delta f(\epsilon)-\cosh ^{2}\left(\frac{\epsilon_{1}}{2 T}\right) \delta f\left(\epsilon_{1}\right) / \cosh ^{2}\left(\frac{\epsilon}{2 T}\right)\right], \\
& K_{22}[\delta f]=-\frac{1}{4} i N(0) g^{2} N_{1}(\epsilon) \int d \Omega_{\hat{p}_{1}} \int \frac{d \epsilon_{1}}{2 \pi}\left[D_{\hat{p} \cdot \hat{p}_{1}}^{R}\left(\epsilon_{1}\right)-D_{\hat{p} \cdot \hat{p}_{1}}\left(\epsilon_{1}\right)\right]\left[\sinh \left(\frac{\epsilon_{1}}{2 T}\right) \cosh \left(\frac{\epsilon_{1}}{2 T}\right)\right]^{-1} \\
& \times\left[\bar{f}_{1}(\epsilon)-\cosh ^{2}\left(\frac{\epsilon_{1}}{2 T}\right) \bar{f}_{1}\left(\epsilon_{1}\right) / \cosh ^{2}\left(\frac{\epsilon}{2 T}\right)\right] .
\end{aligned}
$$

where I have also used the fact that these integrals are practically important only for $\epsilon \leq|\Delta|$, so some of the mild $\epsilon$ dependences in the scale of $T$ may be neglected. To proceed further, we need the behavior of $\delta f$ and $\bar{f}_{1}$ for $\epsilon \sim T$, which may be found selfconsistently. Using Eq. (6.6), or better yet, Eq. (6.11), one can easily conclude that $\delta f \sim \eta^{2}$ for $\epsilon \sim T$, while $\delta f \sim \eta$ for $\epsilon \leq|\Delta|$ (assuming $\tau_{E}^{-1} \sim \eta|\Delta|$. For larger $\tau_{E}^{-1}, \delta f$ is smaller in both energy regions, and becomes unimportant). Thus one may neglect the second term in Eq. (B1), which then reduces to Eq. (6.12). On the other hand, if one wishes to obtain an accurate local charge conservation law, he must preserve the relation $\int_{-\infty}^{\infty} K_{22} d \epsilon=0$, which is true as long as one neglects the second term in Eq. (6.11). This means that the $\bar{f}_{1}\left(\epsilon_{1}\right)$ term in Eq. (B2) should not be neglected, at least not until one invokes the "approximate charge neutrality condition," which is discussed in Sec. X. Another way of seeing this is to realize from Eq. (6.7), or Eq. (6.17), 
that $\bar{f}$ is no larger than $\sim \eta^{2}$ for both $\epsilon \leq|\Delta|$, and $\epsilon \sim T$. To reduce Eq. (B2), therefore, we need a better estimate of $\bar{f}$ for $\epsilon \sim T$, which is obtainable from Eq. (6.7) or Eq. (6.17). Taking this limit, both equations reduce to

$$
\dot{\overline{f_{1}}}-D \nabla^{2} \overline{f_{1}}-e D \vec{\nabla} \cdot \overrightarrow{\mathrm{E}} f_{0, \mathrm{\epsilon}}=K_{22}\left[\overline{f_{1}}\right]
$$

showing that $\bar{f}_{1}=$ const $\times f_{0, \epsilon}$ for $\epsilon \sim T$, where the constant may still depend on $\vec{R}$ and $t$, but it cannot depend on $\epsilon$. Equation (B2) can therefore be reduced to

$$
K_{22}[\delta f]=-\left[N_{1}(\epsilon) / \tau_{E}\right]\left(\overline{f_{1}}-\text { const } \times f_{0, \epsilon}\right) \text {. }
$$

The constant may be determined by the condition $\int_{-\infty}^{\infty} K_{22} d \epsilon=0$. Equation (B3) then reduces to Eq.

The new contributions added to the kinetic equations in Sec. IX do not affect this proof, since they are important only for the energy range $\epsilon \leq|\Delta|$.
'L. P. Gor'kov, Zh. Eksp. Teor. Fiz. 36, 1918 (1959); 37, 1407 (1959) [Sov. Phys. JETP 9, 1364 (1959); 10, 998 (1960)].

${ }^{2}$ V. L. Ginzburg and L. D. Landau, Zh. Eksp. Teor. Fiz. 20, 1064 (1950).

${ }^{3}$ L. P. Gor'kov, Zh. Eksp. Teor. Fiz. 34, 735 (1958) [Sov. Phys. JETP 7, 505 (1958)].

4J. Bardeen, L. N. Cooper, and J. R. Schrieffer, Phys. Rev. 108,1175 (1957).

${ }^{5}$ E. Abrahams and T. Tsuneto, Phys. Rev. 152, 416 (1966).

${ }^{6}$ A. Schmid, Phys. Konden. Mater. 5, 302 (1966).

${ }^{7}$ L. P. Gor'kov and G. M. Éliashberg, Zh. Eksp. Teor. Fiz. 54, 612 (1968) [Sov. Phys. JETP 27, 328 (1968)].

${ }^{8}$ G. M. Éliashberg, Zh. Eksp. Teor. Fiz. 52, 2443 (1968) [Sov. Phys. JETP 28, 1298 (1969)].

${ }^{6}$ C.-R. Hu, Phys. Rev. B 14, 4834 (1976).

${ }^{10}$ A. Schmid and G. Schön, J. Low Temp. Phys. 20, 207 (1975).

${ }^{11}$ A. I. Larkin and Yu. N. Ovchinnikov, Zh. Eksp. Teor. Fiz. 68, 1915 (1975) [Sov. Phys. JETP 41, 960 (1976)].

${ }_{12}$ A. I. Larkin and Yu. N. Ovchinnikov, Zh. Eksp. Teor. Fiz. 73, 299 (1977) [Sov. Phys. JETP 46, 155 (1978)].

${ }_{13} \mathrm{Yu}$. N. Ovchinnikov, J. Low Temp. Phys. 28, 43 (1977).

${ }^{14}$ L. Kramer and R. J. Watts-Tobin, Phys. Rev. Lett. $\underline{40}$, 1041 (1978)

${ }^{15}$ U. Eckern and G. Schön, J. Low Temp. Phys. 32, 821 (1978)

${ }^{16}$ K. D. Usadel, Phys. Rev. Lett. 25, 507 (1970).

${ }^{17} \mathrm{G}$. Schön and V. A mbegaokar, Phys. Rev. B $\underline{19}, 3515$ (1979).

${ }^{18}$ L. V. Keldysh, Zh. Eksp. Teor. Fiz. 47, 1515 (1964) [Sov. Phys. JETP 20, 1018 (1965)].

${ }^{19} \mathrm{G}$. Eilenberger, Z. Phys. 214, 195 (1968).

${ }^{20}$ See, for example V. Ambegaokar, in Superconductivitu. edited by R. D. Parks (Marcel Dekker, New York, 1969), Vol. 1, Chap. 5; and K. Maki, in the same series, Vol. 2 Chap. 18

${ }^{21}$ This cautious statement is made because very close to the gap frequency, $\partial / \partial \epsilon$ is actually of order $(\eta|\Delta|)^{-1}$ corresponding to the smearing of the singularity in the BCS density of state by the depairing mechanisms $\left(\tau_{s}^{-1}, \tau_{E}^{-1}\right.$, or $D k^{2}$ ). If this region is important, then the approximation made in Eq. (5.2) would not be justified. Actually it seems that this region is never really important, as long as some depairing mechanism exists and is of order $\eta|\Delta|$, so we may be on safe ground in using Eq. (4.2). However, a rigorous proof of this statement seems to be difficult.
${ }^{22}$ Equation (5.11) actually follows from the general symmetry relation $\left(\hat{G}_{\epsilon}^{R(\operatorname{or} A)}\right)^{+}=-\tau_{z} \hat{G}_{\epsilon}^{A(\operatorname{or} A)} \tau_{z}$ which may be directly verified by using the definitions [Eqs. (2.1), (2.8), (2.9), (3.4), and (4.1)]. The additional relation $\left(\hat{G}_{\epsilon}^{K}\right)^{+}=+\hat{\tau}_{z} \hat{G}_{\epsilon}^{K} \hat{\tau}_{z}$, in combination with Eq. (3.20), establishes that $\hat{f}^{+}=\hat{f}$, so that $\delta f$ and $f_{1}$ must be real functions.

${ }^{23}$ It should be stressed that $\gamma \equiv 0$ does not imply $\bar{\gamma} \equiv 0$ [cf. Eq. (4.8)], so Eq. (5) of Ref. 14, which is written in a general gauge, is misleading. As a matter of fact, this equation of Ref. 14 should have been written in the real- $\Delta$ gauge, with $2 e \overrightarrow{\mathrm{A}}$ replaced by $-\overrightarrow{\mathrm{Q}}$, and $\Delta$ by $|\Delta|$, in order for it to be consistent with the other equations.

${ }^{24}$ Note that this cancellation would not occur if Eq. (3.21) is modified to include $\hat{\tau}_{x}$ and $\hat{\tau}_{y}$ terms. This hints to the physical soundness in assuming Eq. (3.21), since elastic collisions are expected not to affect the distribution functions.

${ }^{25}$ Notice how the $-i \epsilon \hat{\tau}_{2}$ term of $G^{R}$ (or $A$ ) drops out in the evaluation of $\hat{Z}^{\prime R} \hat{f}-\hat{f} \hat{Z}^{\prime A}$, which would not occur had $\hat{f}$ contained also $\hat{\tau}_{x}$ and $\hat{\tau}_{y}$ terms. Thus even if we do not start with such terms in $\hat{f}$, their kinetic equations will have large coefficients in the homogeneous parts of the two extra inhomogeneous equations, making such terms in $\hat{j}$ very small and negligible. [i.e., $A x=B$ implies $x=B / A$, so $A$ large means $x$ small.] This remark and the one made in the previous footnote support the conclusion that Eq. (3.21) need not be generalized to include $\hat{\tau}_{x}$ and $\hat{\tau}_{y}$ terms.

${ }^{26}$ The order is done as follows: For the energy range $\epsilon \sim T$, Eq. (7.3) implies $\dot{N} \sim \omega \eta^{2} \sim \eta^{3}|\Delta|$, while $f_{1}+e \phi f_{0, \epsilon}$ is no larger than $\eta^{2}$ (see Appendix B), thus the contribution to the integral in Eq. (9.1) from this energy range is no larger than $\eta^{5}|\Delta| \cdot T \sim \eta^{4}|\Delta|^{2}$. On the other hand, for the energy range $\epsilon \sim|\Delta|$, we have $\dot{N} \sim \omega \sim \eta|\Delta|$, while $f_{1}+e \phi f_{0, \epsilon}$ is still no larger than $\eta^{2}$, so the contribution to the integral in Eq. (9.1) from this energy range is no larger than $\eta^{3}|\Delta| \cdot|\Delta| \sim \eta^{3}|\Delta|^{2}$. That $N_{1}, R_{1}, N_{2}$, and $R_{2}$ are all of order 1 for $\epsilon \sim|\Delta|$ may be seen by solving Eq. (5.16) without the left-hand side. [Equation (5.17) then imples $\mathrm{N}_{3} \sim R_{3} \sim \eta$.] It then follows from $\mathrm{Eq}$. (6.17) that $f_{1}-e \phi f_{0, \epsilon} \leq \eta^{2}$ for $\epsilon \leq|\Delta|$, because the term $N_{2}|\Delta|\left(2 f_{1}-\chi f_{0, \epsilon}\right)$ dominates.

${ }^{27} \mathrm{This}$ is obviously not justified for a very narrow range near the gap frequency (i.e., for $|\epsilon-| \Delta|| \leqslant \eta|\Delta|$ ) if $\tau_{s}^{-1} \sim \tau_{E}^{-1} \sim \eta|\Delta|$. However, see the comment in Ref. 21 . 
${ }^{28}$ Remember that the Fermi distribution is related to $f_{0}(\epsilon) \equiv \tanh (\epsilon / 2 T)$ by

$F_{\mathrm{FD}}(\epsilon) \equiv[\exp (\beta \epsilon)+1]^{-1}=\frac{1}{2}\left[1-f_{0}(\epsilon)\right]$.

${ }^{29} \mathrm{This}$ is because $\epsilon$ is measured with respect to the Fermi energy $\epsilon_{F}$, while it is the chemical potential $\mu=\epsilon_{F}+e \phi$, which prefers to take a constant value at equilibrium.

${ }^{30}$ If the phonon or quasiparticle injection is turned on for a while and then turned off, the subsequent behavior of the system can still be described by the present set of equations, if the state right after the injection is known.

${ }^{31}$ Because Kramer and Watts-Tobin have neglected the terms of Eq. (10.8), their expression for $\rho$ is a gross overestimate [Ref. 14, Eq. (14)]. Their solution for
$N_{1}\left(f_{1}+e \phi f_{0, e}\right)$ should be shifted by a nearly constant amount in the whole energy range $\epsilon \leqslant T$, so as to give a much smaller $\int_{0}^{\infty} N_{1}\left(f_{1}+e \phi f_{0, \epsilon}\right) d \epsilon$. This point reveals that the "ultra-smallness" of $\rho$ is not the result of a uniformly small $f_{1}+e \phi f_{0, e}$, but results from a necessary cancellation of the positive and negative contributions in an energy integration. For example, the solution for $f_{1}$ as given by Ref. 14, Eq. (11), is essentially accurate for $\epsilon \leq|\Delta|$, with the approximation considered there. Yet it does not give $\rho$ accurately

${ }^{32}$ See for example, R. S. Thompson and C. -R. Hu, Phys. Rev. Lett. 31, 883 (1973); and Ref. 11. 